ON THE ENERGY OF SUPERCONDUCTORS IN LARGE

AND SMALL DOMAINS

By

\title{
Matthias Kurzke
}

and

\section{Daniel Spirn}

IMA Preprint Series \# 2164

( March 2007)

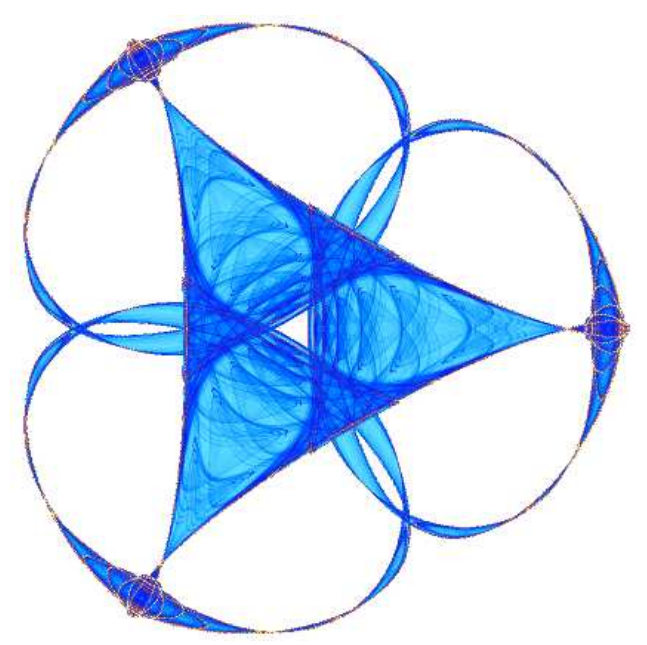

INSTITUTE FOR MATHEMATICS AND ITS APPLICATIONS

UNIVERSITY OF MINNESOTA 400 Lind Hall

207 Church Street S.E.

Minneapolis, Minnesota 55455-0436

Phone: 612-624-6066 Fax: 612-626-7370

URL: http://www.ima.umn.edu 


\title{
ON THE ENERGY OF SUPERCONDUCTORS IN LARGE AND SMALL DOMAINS
}

\author{
MATTHIAS KURZKE AND DANIEL SPIRN
}

\begin{abstract}
We study the Ginzburg-Landau energy functional for superconductors in an applied magnetic field. We focus on asymptotically large or small domains and establish the asymptotic behavior of the energy as a function of the Ginzburg-Landau parameter, applied magnetic field and domain size. For a large class of domain sizes, we calculate the critical field strength where vortex nucleation becomes energetically favorable, and describe the vorticity of minimizers. For supercritical magnetic field strengths, we recover the energy of a classical Abrikosov vortex lattice. Our findings generalize several known results of Sandier and Serfaty for domains of fixed size.
\end{abstract}

\section{Contents}

1. Introduction 1

2. Critical field calculation 8

3. Obstacle problem for small and bounded domains 20

4. Upper bound for vortex lattices 25

5. Lower bound for vortex lattices 30

References $\quad 41$

\section{Introduction}

We study minimizers of the Ginzburg-Landau energy functional

$$
G_{g l}(u, A)=\frac{1}{2} \int_{U_{\ell}}\left|\left(\frac{1}{\kappa} \nabla-i A\right) u\right|^{2}+\left|\operatorname{curl} A-H_{e x}\right|^{2}+\frac{1}{2}\left(1-|u|^{2}\right)^{2} d x
$$

where the domain contains a distinguished length-scale $\ell$. To be precise, we assume that $U \subset \mathbb{R}^{2}$ is a given bounded domain with smooth boundary and $U_{\ell}=\ell U$, a dilation of this domain.

The functional is defined for an order parameter $u: U \rightarrow \mathbb{C}$ whose modulus describes the local density of superconducting Cooper pairs and for a magnetic field potential $A: U \rightarrow \mathbb{R}^{2}$ with $\operatorname{curl} A=h$, the induced magnetic field. The physical parameters in (1.1) are the length scale $\ell$, the applied magnetic field

Date: March 25, 2007.

D. Spirn was supported in part by NSF grant DMS-0510121. 
strength $H_{e x}$, and the Ginzburg-Landau parameter $\kappa$. The two-dimensional energy (1.1) corresponds to superconductors with a cylindrical symmetry and a magnetic field in the perpendicular direction.

Since we are interested in the effect of domain size on the energy of superconducting samples, we scale out the factor $\ell$ and study functionals for various choices of $\ell$ defined over the same domain $U$. Setting $x^{\prime}=\frac{x}{\ell}, \widetilde{u}=u\left(\frac{x}{\ell}\right), \widetilde{A}=\kappa \ell A$, $h_{e x}=\kappa \ell^{2} H_{e x}$, then

$$
G_{g l}(u, A)=\frac{1}{2 \kappa^{2}} \int_{U}\left|\nabla_{\widetilde{A}} \widetilde{u}\right|^{2}+\frac{1}{\ell^{2}}\left|\operatorname{curl} \widetilde{A}-h_{e x}\right|^{2}+\kappa^{2} \ell^{2}\left(1-|\widetilde{u}|^{2}\right)^{2} d x^{\prime} .
$$

Dropping the tildes and primes and using $\varepsilon=\frac{1}{\ell \kappa}$, we are thus lead to study the asymptotics of

$$
G_{\varepsilon}(u, A)=\frac{1}{2} \int_{U}\left|\nabla_{A} u\right|^{2}+\frac{1}{\ell^{2}}\left|\operatorname{curl} A-h_{e x}\right|^{2}+\frac{1}{2 \varepsilon^{2}}\left(1-|u|^{2}\right)^{2} d x
$$

for different choices of $\ell=\ell_{\varepsilon}$, where $\ell_{\varepsilon} \rightarrow \ell_{0} \in[0,+\infty]$ as $\varepsilon \rightarrow 0$. Finally, we can recover the original energy asymptotics

$$
G_{g l}=\frac{G_{\varepsilon}}{\kappa^{2}}
$$

via a change of variables. All results of ours in the following will be stated in terms of $\varepsilon, \ell$, and $h_{e x}$.

We will study type $I I$ superconductors $(\kappa \rightarrow \infty)$. This corresponds to $\varepsilon \rightarrow 0$ if $\ell \ll \frac{1}{\varepsilon}$, which will be implied by other assumptions throughout this article. Under this limit minimizers will start to energetically favor the formation of vortices once the applied magnetic field grows large enough.

The asymptotics of $(1.2)$ with $\ell \equiv 1$, i.e.

$$
\frac{1}{2} \int_{U}\left|\nabla_{A} u\right|^{2}+\left|\operatorname{curl} A-h_{e x}\right|^{2}+\frac{1}{2 \varepsilon^{2}}\left(1-|u|^{2}\right)^{2} d x
$$

under the asymptotic limit $\varepsilon \rightarrow 0$ has been widely studied in the past decade and a half.

1.1. Background results. In the last 15 years, there has been considerable progress made in the mathematical understanding of the Ginzburg-Landau model. A major step has been the groundbreaking work of Bethuel-BrezisHélein [3] on the related functional without gauge term, the so-called BBH functional

$$
E_{\varepsilon}(u)=\frac{1}{2} \int_{U}|\nabla u|^{2}+\frac{1}{2 \varepsilon^{2}}\left(1-|u|^{2}\right)^{2},
$$

and much of the analysis for the full gauge-invariant functional $G_{\varepsilon}$ is based on analysis of $E_{\varepsilon}$. We can only sketch some of the developments for the static Ginzburg-Landau model with magnetic field and refer the reader to the recent 
monograph [15] by Sandier and Serfaty, which contains a thorough treatment of vortex solutions and critical fields for vortex nucleation for (1.3).

We mention some works that are of particular relevance to the topic of this article. The first rigorous treatment of (1.3) in the $\varepsilon \rightarrow 0$ limit can be found in Bethuel-Riviere [4], who discovered many important features of the standard Ginzburg-Landau functional. Serfaty [19, 20] built on this work and gave the first rigorous treatment of the critical field question by a study of local minimizers close to the critical field. The technique used assumptions on the BBH energy (1.4) to obtain an a priori bound on the number of vortices. Using the "vortex ball construction" of Sandier [14] and Jerrard [7], a key ingredient in most of the later research, Sandier-Serfaty [18] were then able to show that the global minimizer below the critical field is indeed vortex-free and $\left|u_{\varepsilon}\right|$ is bounded away from zero.

The structure of global minimizers with an unbounded number of vortices and with external field of order $h_{e x}=O(|\log \varepsilon|)$ was analyzed by Sandier-Serfaty in [17]. This result, combined with a Jacobian compactness theorem [8], was rephrased by Jerrard and Soner [9] in the framework of $\Gamma$-convergence. The limit problem is equivalent to a certain obstacle problem, and the limiting vorticity (after rescaling with $|\log \varepsilon|$ ) is constant in the set where the obstacle is active.

For asymptotically larger applied magnetic fields $\left(|\log \varepsilon| \ll h_{e x} \ll \frac{1}{\varepsilon^{2}}\right)$, the vortices fill the whole domain as an Abrikosov-type lattice with uniform limiting density of vortices. This was established by Sandier-Serfaty [16].

There are few results as of yet on the influence of the domain size on the behavior of the functional. Aftalion and Dancer [1] studied critical points of (1.1). For small domains $\left(\ell<C \min \left(1, \frac{C}{\kappa}\right)\right)$, they showed that any solution that is not the normal solution (where $u \equiv 0$ ) will be bounded away from zero, regardless of the external field. For the special case where the domain is a ball, $U_{\ell}=B_{\ell}(0)$, they showed that solutions in small domains are necessarily radially symmetric, and there exists a critical field of order $O\left(\frac{1}{\ell}\right)$ such that above this field, only the normal solution exists, while a unique superconducting solution exists below this threshold.

A numerical study was performed by Aftalion and Du [2], who studied the response of a superconductor to the raising and lowering of the external field depending on Ginzburg-Landau parameter $\kappa$ and domain size. They found bifurcation diagrams in several distinct regimes, including a critical line separating type I and type II behavior.

There have also been a few results that study (1.3) with applied magnetic fields and domain dependence between $h_{c_{2}}$ and $h_{c_{3}}$, the regime associated with surface superconductivity. However, we restrict ourselves to field strengths asymptotically below $h_{c_{2}}$, hence we do not attempt to review results within this class of field strengths. 
There are similarities between the Ginzburg-Landau energy (1.2) and the Chern-Simons-Higgs energy

$$
G_{c s h}(u, A)=\frac{1}{2} \int_{U}\left|\nabla_{A} u\right|^{2}+\frac{\mu^{2}}{4} \frac{\left|\operatorname{curl} A-h_{e x}\right|^{2}}{|u|^{2}}+\frac{1}{\varepsilon^{2}}|u|^{2}\left(1-|u|^{2}\right)^{2} d x
$$

for an applied magnetic field, $h_{e x}$, and a bounded, simply connected domain, $U \subset \mathbb{R}^{2}$. The Chern-Simons-Higgs model is an anyon theory that is of interest in connection with high-temperature superconductors and the quantum Hall effect. For an overview of the study in the self-dual case $\mu=\varepsilon$, see Yang [22].

For $|u| \leq 1$ with $|u| \approx 1$ on $\partial U$, the authors proved several results of a similar nature to those found here: For $h_{e x}=H|\log \varepsilon|$ and $G_{c s h}\left(u_{\varepsilon}, A_{\varepsilon}\right)=O\left(|\log \varepsilon|^{2}\right)$, we were able to show $\Gamma$-convergence results for the cases $\mu=\mu_{\varepsilon} \rightarrow \mu_{0} \in(0, \infty]$, see $[11,12]$. These enabled us to calculate the critical field for vortex nucleation. The main ingredient in these results is a compactness proof that relates the Jacobian of $u, J(u)=\operatorname{det} \nabla u$, to the energy

$$
E_{c s h}(u)=\frac{1}{2} \int_{U}|\nabla u|^{2}+\frac{1}{\varepsilon^{2}}|u|^{2}\left(1-|u|^{2}\right) .
$$

Using this compactness result from [11] and an energy decomposition, we showed $\Gamma$-convergence for finite $\mu$ in [11] and for $\mu \rightarrow \infty$ in [12]. For $\mu \rightarrow 0$, we gave an explicit counterexample that illustrates why this method, using a decomposition and bounds for $E_{c s h}$, fails in this case. However, we were later able to show that for $h_{e x}$ much larger than the critical field, and under certain restrictions on $\mu$, the energy of minimizers scale in the same fashion as the energy of an Abrikosov type lattice just as for the Ginzburg-Landau energy (1.3), see [13].

All of our results here carry over from the Chern-Simons-Higgs energy (1.5) under the assumptions above. In particular, we can extend the results of our previous articles $[11,12,13]$ and understand vortices in non-selfdual CSH for a wider range of parameters and in more detail. Results for (1.2) in the next subsection can be related to results for (1.5) by simply setting $\ell=\frac{2}{\mu}$.

1.2. Main results. In this subsection, we list our main theorems on the behaviour of minimizers for various parameter regimes. These results, most of which are generalizations of known results from the last section, provide a partial solution to Open Problem 1 of [15].

Our first result is the calculation of the first critical field where minimizers of the Ginzburg-Landau energy start to have vortices. This field is $O(|\log \varepsilon|)$ if the domains stay bounded and $O\left(\ell^{2}|\log \varepsilon|\right)$ if the domains are unbounded and $\ell$ is bounded by a power of $|\log \varepsilon|$ :

Theorem 1.1. There exists a sequence of critical fields $h_{c_{1}}(\varepsilon)$ such that any minimizer of the Ginzburg-Landau functional with $h_{e x}<h_{c_{1}}(\varepsilon)-o(|\log \varepsilon|)$ is vortex-free, while any minimizer with $h_{e x}>h_{c_{1}}(\varepsilon)+o(|\log \varepsilon|)$ has vortices. 
As $\varepsilon \rightarrow 0$, the critical field $h_{c_{1}}(\varepsilon)$ satisfies the following expansion: If $\ell_{\varepsilon} \rightarrow \ell_{0}$ with $0 \leq \ell_{0}<\infty$, then

$$
\frac{h_{c_{1}}(\varepsilon)}{|\log \varepsilon|} \rightarrow H_{1}\left(\ell_{0}\right)
$$

where

$$
H_{1}\left(\ell_{0}\right)=\frac{1}{2 \max _{\bar{U}}\left|y_{\ell_{0}}\right|},
$$

where $y_{\ell_{0}}$ is the solution of

$$
-\Delta y_{\ell_{0}}+\ell_{0}^{2} y_{\ell_{0}}+1=0
$$

with Dirichlet boundary conditions $y_{\ell_{0}}=0$ on $\partial U$.

Finally, if $\ell_{\varepsilon} \rightarrow \infty$ and $\ell_{\varepsilon} \leq|\log \varepsilon|^{\gamma}$ for any fixed $\gamma>0$, then

$$
\frac{h_{c_{1}}(\varepsilon)}{\ell_{\varepsilon}^{2}|\log \varepsilon|} \rightarrow \frac{1}{2}
$$

as $\varepsilon \rightarrow 0$. Therefore, the critical field scales as $h_{c_{1}}=\frac{\ell_{\varepsilon}^{2}|\log \varepsilon|}{2}$ in this regime of domain sizes.

For small or bounded domains Theorem 1.1 follows from adapting the proof of Sandier and Serfaty [18], where $\ell \equiv 1$. Formally examining the resulting critical field (1.7), one finds $H_{1}\left(\ell_{0}\right) \rightarrow \frac{\ell_{0}^{2}}{2}$ as $\ell_{0} \rightarrow \infty$, so we expect that $h_{c_{1}}=\frac{\ell_{\varepsilon}^{2}|\log \varepsilon|}{2}$ for any $\ell_{\varepsilon} \rightarrow+\infty$ and $\ell_{\varepsilon} \ll \frac{1}{\sqrt{\varepsilon}|\log \varepsilon|}$, see the discussion before Lemma 2.7. We give a proof for the case of large (but not too large) domains in Section 2, see Proposition 2.1 for details.

The following results can be used to characterize the minimizers of $\left(u_{\varepsilon}, A_{\varepsilon}\right)$ for external fields of order $O(|\log \varepsilon|)$ and small or bounded domains. The first step is a $\Gamma$-convergence result that relates $G_{\varepsilon}\left(u_{\varepsilon}, A_{\varepsilon}\right)$ to a simpler functional that no longer involves $\varepsilon$. We skip some of the detailed convergence statements for ease of presentation. The full statement is given in Theorem 3.1.

Theorem 1.2. As $\varepsilon \rightarrow 0$, the functional $\frac{1}{|\log \varepsilon|^{2}} G_{\varepsilon}$ is $\Gamma$-convergent to $G(v, a)$, where the limit functional $G$ is given by

$$
\begin{aligned}
& G(v, a):= \\
& \begin{cases}\frac{1}{2} \int_{U}|v-a|^{2}+\frac{1}{\ell_{0}^{2}}|\operatorname{curl} a-H|^{2}+\frac{1}{2}\|\operatorname{curl} v\|_{\mathscr{M}} & \text { if } \ell_{0}>0 \\
\frac{1}{2} \int_{U}|v-a|^{2}+\frac{1}{2}\|\operatorname{curl} v\|_{\mathscr{M}} & \text { if } \ell_{0}=0 \text { and } \operatorname{curl} a=H \\
+\infty & \text { else, }\end{cases}
\end{aligned}
$$

under a convergence that includes $\left(\frac{1}{|\log \varepsilon|}\left(i u_{\varepsilon}, \nabla u_{\varepsilon}\right)-\frac{1}{|\log \varepsilon|}|u|^{2} A_{\varepsilon}\right) \rightarrow(v-a)$ and $\frac{1}{|\log \varepsilon|} A_{\varepsilon} \rightarrow \operatorname{curl} a$ in $L^{2}$. 
Since $\Gamma$-convergence and the compactness we have here imply that minimizers of $G_{\varepsilon}$ and of $G$ approximate each other, we study minimizers of $G$ to gain insight into the structure of minimizers of $G_{\varepsilon}$.

Theorem 1.3. If $\left(v_{0}, a_{0}\right)$ is a minimizer of (1.9) and $\ell_{0}>0$, then $z_{0}=$ $\ell_{0}^{-2}\left(\operatorname{curl} a_{0}-H\right)$ is the unique minimizer of the following obstacle problem: Minimize

$$
F_{\ell_{0}, H}(z)=\frac{1}{2} \int_{U}|\nabla z|^{2}+\ell_{0}^{2} z^{2}+2 z H
$$

in the admissible class

$$
\mathscr{K}=\left\{z \in H_{0}^{1}(U): z \geq-\frac{1}{2} \text { a.e. in } U\right\} .
$$

The limit $\left(v_{0}, a_{0}\right)$ satisfies the following additional properties:

$$
\begin{aligned}
\ell_{0}^{-2} \operatorname{curl}\left(\operatorname{curl} a_{0}-H\right)+a_{0} & =v_{0} \quad \text { in } U \\
\operatorname{curl} a_{0}-H & =0 \quad \text { on } \partial U \\
-\frac{1}{2} & \leq z_{0} \leq 0 \\
\operatorname{curl} v_{0} & \geq 0 \\
\operatorname{spt}\left(\operatorname{curl} v_{0}\right) & \subset\left\{z_{0}=-\frac{1}{2}\right\} .
\end{aligned}
$$

In the case where $\ell_{0}=0$, we have curl $a_{0}=H$, and obtain a slightly different obstacle problem: Let $y_{0}$ be the solution of $-\Delta y_{0}=\operatorname{curl} v_{0}-H$ with zero boundary conditions. Then $y_{0}$ is the unique minimizer of

$$
F_{0, H}(y)=\frac{1}{2} \int_{U}|\nabla y|^{2}+2 y H
$$

in the admissible class

$$
\mathscr{K}=\left\{y \in H_{0}^{1}(U): y \geq-\frac{1}{2} \text { a.e. in } U\right\} .
$$

Moreover, $\operatorname{curl} v_{0} \geq 0$, and $\operatorname{spt}\left(\operatorname{curl} v_{0}\right) \subset\left\{y_{0}=-\frac{1}{2}\right\}$.

This theorem, proved later as Theorem 3.3, implies again the results on the first critical field: When the obstacle is not active, the minimizer satisfies curl $v=0$. This happens if and only if $H<H_{1}\left(\ell_{0}\right)$ with the same fields as above. However, since we rescaled the vorticity to obtain convergence, this only shows that an approximating sequence $\left(u_{\varepsilon}, A_{\varepsilon}\right)$ has at most $o(|\log \varepsilon|)$ vortices, a result that is weaker than the "no vortices below the critical field" obtained in Theorem 1.1.

Finally, we study minimizers of the Ginzburg-Landau functional with a very large (supercritical) applied external magnetic field and obtain energy asymptotics of a uniform vortex lattice: 
Theorem 1.4. Assume that

$$
\max \left\{1, \ell^{2}\right\}|\log \varepsilon| \ll h_{e x} \ll \frac{1}{\varepsilon^{2}},
$$

then minimizing sequence $\left\{u_{\varepsilon}, A_{\varepsilon}\right\}$ satisfies:

$$
G_{\varepsilon}\left(u_{\varepsilon}, A_{\varepsilon}\right)=\frac{1}{2}|U| h_{e x} \log \frac{1}{\varepsilon \sqrt{h_{e x}}}\left(1-o_{\varepsilon}(1)\right) .
$$

Furthermore, the vortex density is uniform in the limit, see Proposition 5.3 for a precise statement.

The proof of Theorem 1.4 is divided into an upper bound Proposition 4.1 and a lower bound part Proposition 5.1. For the lower bound, we follow SandierSerfaty [16] using employing the co-area formula with the vortex-ball method; however, we use a more careful estimates that refines a lower bound on the magnetic field part of the energy term. This allows us to establish the asymptotics for the full range of supercritical applied magnetic fields. In particular our lower bound depends on lower bound Lemma 5.6 and Lemma 5.7 that takes into account the length scale $\ell$ versus the size of the vortex ball $r$. Such care is not so important in the $\ell \equiv 1$ case, but crucial in the $\ell \rightarrow \infty$ case.

We compute the upper bound with a simplified approach using Fourier series (Proposition 4.1). A refined version of the upper bound, (4.2), motivates our conjecture on the behavior close to the critical field for large domains.

Remark 1.5. For $\ell_{\varepsilon} \rightarrow \infty$ and $h_{e x}=O\left(\ell^{2}|\log \varepsilon|\right)$, we do not yet have a rigorous result on the structure of minimizers. However, we expect from formal calculations that a uniform lattice, as those constructed in Section 4, should be minimizing.

1.3. Discussion. We conclude the introduction with several unresolved questions regarding asymptotics of (1.2). There is still work to do to complete the answer to Problem 1 in [15]. In particular, a complete phase diagram for the minimizing behavior depending on $\kappa, \ell$, and $h_{e x}$ should be given, including the cross-over between type I and type II behavior that happens for $\kappa \ell=O(1)$, and the results of Aftalion and $\mathrm{Du}[2]$ should be made fully rigorous. For such a study, it would also be necessary to understand local minimizers and hysteresis phenomena for slowly changing fields.

It is an interesting problem to further study beginning vortex nucleation close to the critical field in the large domain limit, $\ell \rightarrow+\infty$. Based on the construction of Proposition 4.1 and the structure of the Meissner state, we expect that minimizers exhibit a uniform vortex lattice that fills the whole domain. However, vortices will be far apart and interact only weakly, making this a subtle problem. Finally, it would be interesting to study (1.2) with applied fields in the "intermediate range", recently undertaken for (1.3) in [15]. 
For states with few vortices in large domains, we similarly expect very slow motion for the gradient flow, as vortices will move in an almost flat potential.

\section{Critical field CAlculation}

In this section we establish Theorem 1.1. The proof of Theorem 1.1 for $\ell \rightarrow[0,+\infty)$ follows from a direct insertion of $\ell^{-2}$ in the magnetic field term of (1.3) and following the proof found in [19, 18]. However, when $\ell \rightarrow+\infty$, a simple scaling argument fails, and we need to be more careful. In the following we show that for a substantial class of large-domain asymptotics, the critical field strength is indeed

$$
h_{c_{1}}=\frac{\ell^{2}}{2}|\log \varepsilon|+o(|\log \varepsilon|),
$$

as suggested by the formal analysis of the scaled renormalized energy.

Proposition 2.1. Let $\ell \rightarrow+\infty$ with $\ell \leq C|\log \varepsilon|^{\gamma}$ for any fixed $\gamma \in \mathbb{R}^{+}$and suppose $(u, A)$ is a minimizer over the Ginzburg-Landau energy (1.2). Then the first critical field for vortex nucleation is $h_{c_{1}}=\frac{\ell^{2}}{2}|\log \varepsilon|=\frac{1}{2}|\log \varepsilon|^{2 \gamma+1}$. In particular for $h_{e x}<\frac{\ell^{2}}{2}|\log \varepsilon|$, any minimizer will be satisfy $|u| \geq \frac{3}{4}$ for all $\varepsilon$ sufficiently small, and for $h_{e x}>\frac{\ell^{2}}{2}|\log \varepsilon|$ any minimizer must have a vortex.

Remark 2.2. Although we establish the conjectured critical field for $\ell=|\log \varepsilon|^{\gamma}$, we believe the critical field should be true over length scales up to $\ell \leq \frac{C}{\sqrt{\varepsilon}|\log \varepsilon|}$. In particular, the more refined vortex ball estimates found in $[10,15]$ should be powerful enough to handle larger domains, but in the interest of brevity we consider only $\ell$ 's of the form $\ell=C|\log \varepsilon|^{\gamma}$. We establish Proposition 2.1 by using the explicit vortex structure that exists for these intermediate-sized domains.

The Euler-Lagrange equations of (1.2)

$$
\begin{aligned}
& 0=\nabla_{A}^{2} u+\frac{1}{\varepsilon^{2}} u\left(1-|u|^{2}\right) \\
& 0=\operatorname{curl} \operatorname{curl} A+\ell^{2} j_{A}(u)
\end{aligned}
$$

in $U$ and $n \cdot \nabla_{A} u=0$ and $\operatorname{curl} A=h_{e x}$ on $\partial U$. Setting the Coulomb gauge we see that

$$
\operatorname{div} A=0 \text { in } U \quad n \cdot A=0 \text { on } \partial U .
$$

Solutions to (2.1) satisfy the maximum principle

$$
\|u\|_{L^{\infty}(U)} \leq 1
$$

the proof of which can be found in $[4,16]$. 
The key to establishing Proposition 2.1 is a good energy decomposition. In order to establish this decomposition we use the following result of SandierSerfaty that supplies the vortex structure for our range of $\ell$ 's. Their result, based on the method of Jerrard [7] and Jerrard-Soner [8] is:

Proposition 2.3 (Sandier-Serfaty[18]). Let $u: U \rightarrow \mathbb{C}$ be such that $|\nabla u| \leq \frac{C}{\varepsilon}$ and that $E_{\varepsilon}(u) \leq C|\log \varepsilon|^{M}$ for $M \geq 2$ a fixed number. Then, for any $\alpha>0$ there exists disjoint balls $\left\{B_{r_{i}}\right\}_{i \in I}$ of radii $r_{i}$ such that for sufficiently small $\varepsilon$,

(1) $\left\{|u|<\frac{3}{4}\right\} \subset \cup_{i \in I} B_{r_{i}}$

(2) $\operatorname{card} I \leq C|\log \varepsilon|^{M}$

(3) $r_{i} \leq \frac{C}{|\log \varepsilon|^{\alpha}}$

(4) if $\overline{B_{r_{i}}} \subset U$ and $d_{i}=\operatorname{deg}\left(u, \partial B_{r_{i}}\right)$, then

$$
\int_{B_{r_{i}}} e_{\varepsilon}(u) d x \geq \pi\left|d_{i}\right|(|\log \varepsilon|-O(\log |\log \varepsilon|)) .
$$

Remark 2.4. The result in [18] is restricted to energies of the size $E_{\varepsilon}(u) \leq$ $K|\log \varepsilon|^{2}$; however, the same proof holds for the higher energies in the assumptions found in Proposition 2.1.

We now state our energy decomposition, in the spirit of Bethuel-Riviere, Serfaty, and Sandier-Serfaty, [4, 19, 16].

Proposition 2.5. Let $(u, A)$ be a minimizer where $A$ satisfies the Coulomb gauge and $\ell=C|\log \varepsilon|^{\gamma}$. Let $A=h_{e x} \nabla^{\perp} \xi_{\ell}+\nabla^{\perp} \zeta$ where $\xi_{\ell}$ satisfies

$$
\begin{aligned}
-\frac{1}{\ell^{2}} \Delta^{2} \xi_{\ell}+\Delta \xi_{\ell} & =0 \text { in } U \\
\Delta \xi_{\ell} & =1 \text { on } \partial U \\
\xi_{\ell} & =0 \text { on } \partial U
\end{aligned}
$$

then

$$
\begin{aligned}
G_{\varepsilon}(u, A) \geq & \sum_{i \in I} \int_{B_{r_{i}}} e_{\varepsilon}(u) d x+\frac{1}{\ell^{2}} \int_{U}|\Delta \zeta|^{2}+G^{0} \\
& +2 \pi h_{e x} \sum_{i \in I} d_{i} \xi_{\ell}\left(a_{i}\right)-\frac{C}{|\log \varepsilon|}
\end{aligned}
$$

where $G^{0}=G_{\varepsilon}\left(1, h_{e x} \nabla^{\perp} \xi_{\ell}\right)$. Here the vortex balls $B_{r_{i}}$ and degrees $d_{i}$ are defined via Proposition 2.3

We prove several intermediate lemmas before attempting the proof of Proposition 2.5. The first facts we establish are on the scaled London equation. This limiting equation for the stream function of the magnetic field potential is the expected Meissner solution. 
Lemma 2.6. Let $\xi_{\ell}$ be a solution of (2.4) with $\ell \gg 1$, then

$$
-\frac{1}{\ell^{2}} \leq \xi_{\ell} \leq 0
$$

and

$$
\sup _{x \in \bar{U}}\left|\xi_{\ell}\right|=\frac{1}{\ell^{2}}\left(1-o_{\ell}(1)\right)
$$

Further,

$$
\left\|\xi_{\ell}\right\|_{H^{2}} \leq C \quad \text { and } \quad\left\|\nabla \xi_{\ell}\right\|_{L^{\infty}} \leq C
$$

where $C$ depends only on $U$.

Proof. These results are similar to results found in $[4,19,21]$ for $\ell \equiv 1$. If $\Delta \xi_{\ell}=h_{\ell}$ in $U$ and $\xi_{\ell}=0$ on $\partial U$, then $h_{\ell}$ satisfies

$$
\begin{aligned}
-\frac{1}{\ell^{2}} \Delta h_{\ell}+h_{\ell} & =0 \text { in } U \\
h_{\ell} & =1 \text { on } \partial U .
\end{aligned}
$$

If we let $\chi=\xi_{\ell}-\frac{1}{\ell^{2}}\left(h_{\ell}-1\right)$, then $\Delta \chi=\Delta \xi_{\ell}-\frac{1}{\ell^{2}} \Delta\left(h_{\ell}-1\right)=h_{\ell}-\frac{1}{\ell^{2}} \Delta h_{\ell}=0$ in $U$ and $\chi=0$ on $\partial U$. Therefore, $\chi \equiv 0$, thus

$$
\xi_{\ell}=\frac{1}{\ell^{2}}\left(h_{\ell}-1\right) \text {. }
$$

Applying the maximum principle to (2.9) yields $0<h_{\ell}<1$. In particular if a minimum occurs at a point $x_{m}$ in the interior of $U$ then $0<\frac{1}{\ell^{2}} \Delta h_{\ell}\left(x_{m}\right)=$ $h_{\ell}\left(x_{m}\right)$, and by the boundary condition we see $h_{\ell} \geq 0$. On the other hand if the maximum occurs at a point $x_{M}$ in the interior of $U$ then $0>\frac{1}{\ell^{2}} \Delta h_{\ell}\left(x_{M}\right)=$ $h_{\ell}\left(x_{M}\right)$, and by the boundary condition $h_{\ell} \leq 1$. Applying this to (2.10) yields $(2.6)$.

Next, using the boundary conditions on $\xi_{\ell}$,

$$
\begin{aligned}
0 & =\int_{U} \xi_{\ell}\left[-\frac{1}{\ell^{2}} \Delta^{2} \xi_{\ell}+\Delta \xi_{\ell}\right]=\int_{U} \frac{1}{\ell^{2}} \nabla \xi_{\ell} \cdot \nabla \Delta \xi_{\ell}-\left|\nabla \xi_{\ell}\right|^{2} \\
& =\int_{\partial U} \frac{1}{\ell^{2}} \partial_{n} \xi-\int_{U}\left[\frac{1}{\ell^{2}}\left|\Delta \xi_{\ell}\right|^{2}+\left|\nabla \xi_{\ell}\right|^{2}\right]
\end{aligned}
$$

thus by (2.9), (2.10), and the bound on $h_{\ell}$,

$$
\int_{U} \frac{1}{\ell^{2}}\left|\Delta \xi_{\ell}\right|^{2}+\left|\nabla \xi_{\ell}\right|^{2}=\frac{1}{\ell^{2}} \int_{U} \Delta \xi_{\ell}=\frac{1}{\ell^{2}} \int_{U} h_{\ell} \leq \frac{1}{\ell^{2}}|U| .
$$

This implies $\left\|\xi_{\ell}\right\|_{H^{2}(U)} \leq 2 \sqrt{|U|}$ and (2.8) by Sobolev embedding.

Set $z_{\ell}=\partial_{\ell} h_{\ell}$ then $z_{\ell}$ satisfies

$$
\Delta z_{\ell}-\ell^{2} z_{\ell}=2 \ell h_{\ell} \geq 0
$$


in $U$ and $z_{\ell}=0$ on $\partial U$. By the maximum principle $z_{\ell} \leq 0$; hence, $h_{\ell}$ is monotonically decreasing in $\ell$ for all $x \in U$. Since $h_{\ell}$ is bounded below by -1 then $h_{\ell}(x)=-1+o_{\ell}(1)$ for all $x \in U^{\prime} \Subset U$. Thus $\max \left|\xi_{\ell}\right|=\frac{1}{\ell^{2}}\left(1-o_{\ell}(1)\right)$ by (2.10).

In order to use Proposition 2.3 we need to establish a bound on $E_{\varepsilon}(u)$, see (1.4). As we see below, the BBH energy can be much larger than the GinzburgLandau energy $G_{\varepsilon}(u, A)$, since the magnetic field term in the energy can absorb large induced fields, generated by a large number of vortices. We have

Lemma 2.7. Let $(u, A)$ be a minimizer of the Ginzburg-Landau energy. Suppose $h_{e x} \leq C \ell^{2}|\log \varepsilon| \leq \frac{C}{\varepsilon}$ and $G_{\varepsilon}(u, A) \leq K \ell^{2}|\log \varepsilon|^{2}$ then

$$
E_{\varepsilon}(u) \leq C \ell^{4}|\log \varepsilon|^{2}
$$

and

$$
\|A\|_{H^{1}(U)} \leq C \ell^{2}|\log \varepsilon| \quad \text { and } \quad\|A\|_{H^{2}(U)} \leq C \ell^{3}|\log \varepsilon| .
$$

Proof. We first establish a uniform $H^{1}$ estimate on $A$. From the assumption on the energy

$$
\int\left|h-h_{e x}\right|^{2} \leq K \ell^{4}|\log \varepsilon|^{2}
$$

hence from the bound on $h_{e x}$ we see that

$$
\|h\|_{L^{2}(U)} \leq C \ell^{2}|\log \varepsilon|
$$

Since $\operatorname{div} A=0$ and $n \cdot A=0$ on $\partial U$, there exists $\xi$ such that $\nabla^{\perp} \xi=A$ and $\xi=0$ on $\partial U$. From standard elliptic estimates we get $\|\xi\|_{H^{2}(U)} \leq C \ell^{2}|\log \varepsilon|$. Thus

$$
\|A\|_{H^{1}(U)} \leq C \ell^{2}|\log \varepsilon|
$$

Decomposing $\frac{1}{2}\left|\nabla_{A} u\right|^{2}=\frac{1}{2}|\nabla u|^{2}-A \cdot j(u)+\frac{1}{2} A^{2}|u|^{2}$, we control the cross term via

$$
\begin{aligned}
A \cdot j(u) & \leq \frac{1}{4}\left|\frac{j(u)}{|u|}\right|^{2}+A^{2}|u|^{2} \\
& \leq \frac{1}{4}\left|\frac{j(u)}{|u|}\right|^{2}+A^{2}+2 \varepsilon^{2} A^{4}+\frac{1}{8 \varepsilon^{2}}\left(1-|u|^{2}\right)^{2} .
\end{aligned}
$$


Therefore, from the algebraic bounds, the estimate on $A$, and Sobolev embedding

$$
\begin{aligned}
G_{\varepsilon}(u, A) & \geq E_{\varepsilon}+\frac{1}{2 \ell^{2}}\left\|h-h_{e x}\right\|^{2}-\left[\frac{1}{2} E_{\varepsilon}(u)+\int_{U} A^{2}+2 \varepsilon^{2} A^{4}\right] \\
& \geq \frac{1}{2} E_{\varepsilon}(u)-C\left[\|A\|_{H^{1}(U)}^{2}+\varepsilon^{2}\|A\|_{H^{1}(U)}^{4}\right] \\
& \geq \frac{1}{2} E_{\varepsilon}(u)-C \ell^{4}|\log \varepsilon|^{2}-C \varepsilon^{2} \ell^{8}|\log \varepsilon|^{4} .
\end{aligned}
$$

The upper bound on $E_{\varepsilon}(u)$ follows.

In order to establish higher bounds on $A$ we use the Euler-Lagrange equation $-\nabla^{\perp} h=\ell^{2} j_{A}(u)$. Therefore,

$$
\|\nabla h\|_{L^{2}(U)} \leq \ell^{2}\left\|\nabla_{A} u\right\|_{L^{2}(U)}\|u\|_{L^{\infty}(U)} \leq C \ell^{3}|\log \varepsilon|,
$$

and hence (2.8).

The fact that $E_{\varepsilon}(u)$ can have a much larger energy than $G_{\varepsilon}(u, A)$ is an essential difference in the large $\ell$ asymptotics. It implies a more complicated global vortex structure. Given the energy bound on $E_{\varepsilon}(u)$, we can split apart the full Ginzburg-Landau energy into its chief components. We start with an initial energy splitting.

Lemma 2.8. We can decompose $G_{\varepsilon}(u, A)=G_{\varepsilon}\left(u, \nabla^{\perp} \xi\right)=G_{\varepsilon}\left(u, h_{e x} \nabla^{\perp} \xi_{\ell}+\right.$ $\left.\nabla^{\perp} \zeta\right)$ as

$$
\begin{aligned}
G_{\varepsilon}(u, A) \geq & \frac{1}{2} \int_{U}\left|\nabla u-i u \nabla^{\perp} \xi\right|^{2}+\frac{1}{2 \varepsilon^{2}}\left(1-|u|^{2}\right)^{2}+\frac{1}{2 \ell^{2}} \int_{U}|\Delta \xi|^{2} \\
& +\int_{U}\left(\nabla u,-i h_{e x} \nabla^{\perp} \xi_{\ell} u\right)+G^{0}-\frac{C}{|\log \varepsilon|}
\end{aligned}
$$

Proof. We decompose the energy in a series of steps.

Our first step is to compute the approximate energy of the Meissner state via the method of Serfaty [19]. Since $\operatorname{div} A=0$ and $n \cdot A=0$ in $\partial U$ then we can write $A=\nabla^{\perp} \xi$ with $\xi=0$ on $\partial U$ and so $\Delta \xi=h$. We further decompose $\nabla^{\perp} \xi$ as $\nabla^{\perp} \xi=h_{e x} \nabla^{\perp} \xi_{\ell}+\nabla^{\perp} \zeta$ where $\zeta=\Delta \zeta=0$ on $\partial U$ and where $\xi_{\ell}$ satisfies (2.4). Consider now the Meissner energy associated to $G^{0}=G_{\varepsilon}\left(1, h_{e x} \nabla^{\perp} \xi_{\ell}\right)$. We compute the form of the Meissner energy, setting $\left(u_{0}, A_{0}\right)=\left(1, h_{e x} \nabla^{\perp} \xi_{\ell}\right)$. Then

$$
\begin{aligned}
G_{\varepsilon}\left(u_{0}, A_{0}\right) & =\frac{1}{2} \int_{U}\left|\nabla_{A_{0}} u_{0}\right|^{2}+\frac{1}{\ell^{2}}\left|\operatorname{curl} A_{0}-h_{e x}\right|^{2}+\frac{1}{2 \varepsilon^{2}}\left(1-\left|u_{0}\right|^{2}\right)^{2} \\
& =\frac{h_{e x}^{2}}{2} \int_{U}\left|\nabla \xi_{\ell}\right|^{2}+\frac{1}{\ell^{2}}\left|\Delta \xi_{\ell}-1\right|^{2}
\end{aligned}
$$


Multiplying $\xi_{\ell}$ against $-\frac{1}{\ell^{2}} \Delta^{2} \xi_{\ell}+\Delta \xi_{\ell}$ and integrating over $U$ yields

$$
\int_{U}\left|\nabla \xi_{\ell}\right|^{2}+\frac{1}{\ell^{2}}\left|\Delta \xi_{\ell}\right|^{2} d x=\frac{1}{\ell^{2}} \int_{U} \Delta \xi_{\ell} .
$$

We use the above identity to rewrite the Meissner energy as

$$
\begin{aligned}
G^{0} & =\frac{1}{2} \int_{U} h_{e x}^{2}\left|\nabla \xi_{\ell}\right|^{2}+\frac{h_{e x}^{2}}{\ell^{2}}\left|\Delta \xi_{\ell}\right|^{2}+\frac{h_{e x}^{2}}{\ell^{2}}-2 \frac{h_{e x}^{2}}{\ell^{2}} \Delta \xi_{\ell} \\
& =-\frac{h_{e x}^{2}}{2} \int_{U}\left[\left|\nabla \xi_{\ell}\right|^{2}+\frac{1}{\ell^{2}}\left|\Delta \xi_{\ell}\right|^{2}\right]+\frac{h_{e x}^{2}}{2 \ell^{2}}|U| .
\end{aligned}
$$

Therefore the Meissner energy is of order $O\left(\frac{h_{e x}^{2}}{\ell^{2}}\right)$.

Next we write

$$
\begin{aligned}
\frac{1}{2} \int_{U}\left|\nabla_{A} u\right|^{2}= & \frac{1}{2} \int_{U}\left|\nabla u-i h_{e x} \nabla^{\perp} \xi_{\ell} u-i \nabla^{\perp} \zeta u\right|^{2} \\
= & \frac{1}{2} \int_{U}\left|\nabla u-i \nabla^{\perp} \zeta u\right|^{2}+\frac{h_{e x}^{2}}{2} \int_{U}|u|^{2}\left|\nabla^{\perp} \xi_{\ell}\right|^{2}+ \\
& +\int_{U}\left(\nabla u-i \nabla^{\perp} \zeta u,-i h_{e x} \nabla^{\perp} \xi_{\ell} u\right)
\end{aligned}
$$

and

$$
\begin{aligned}
\int_{U}( & \left.\nabla u-i \nabla^{\perp} \zeta u,-i h_{e x} \nabla^{\perp} \xi_{\ell} u\right) \\
& =\int_{U}\left(\nabla u,-i h_{e x} \nabla^{\perp} \xi_{\ell} u\right)+h_{e x} \int_{U}|u|^{2} \nabla \xi_{\ell} \cdot \nabla \zeta .
\end{aligned}
$$

Therefore, we can write the Ginzburg-Landau energy as

$$
\begin{aligned}
G_{\varepsilon}(u, A)= & \frac{1}{2} \int_{U}\left|\nabla u-i \nabla^{\perp} \zeta u\right|^{2}+\frac{1}{2 \varepsilon^{2}}\left(1-|u|^{2}\right)^{2} \\
& +\int_{U}\left(\nabla u,-i h_{e x} \nabla^{\perp} \xi_{\ell} u\right) \\
& +\frac{h_{e x}^{2}}{2} \int_{U}\left(|u|^{2}-1\right)\left|\nabla^{\perp} \xi_{\ell}\right|^{2}+h_{e x} \int_{U}\left(|u|^{2}-1\right) \nabla \xi_{\ell} \cdot \nabla \zeta \\
& +\frac{1}{2 \ell^{2}} \int_{U}\left|h-h_{e x}\right|^{2}+\frac{h_{e x}^{2}}{2} \int_{U}\left|\nabla^{\perp} \xi_{\ell}\right|^{2}+h_{e x} \int_{U} \nabla \xi_{\ell} \cdot \nabla \zeta .
\end{aligned}
$$

The terms in the third line of (2.15) are small since

$$
\begin{aligned}
h_{e x}^{2} \int_{U}\left(|u|^{2}-1\right)\left|\nabla \xi_{\ell}\right|^{2} & \leq C h_{e x}^{2}\left\|\nabla \xi_{\ell}\right\|_{L^{\infty}}^{2}\left\|1-|u|^{2}\right\|_{L^{2}} \leq C \varepsilon h_{e x}^{2} E_{\varepsilon}^{\frac{1}{2}}(u) \\
& \leq C \varepsilon \ell^{6}|\log \varepsilon|^{3} \leq C \varepsilon|\log \varepsilon|^{6 \gamma+3} \leq \frac{C}{|\log \varepsilon|}
\end{aligned}
$$


and

$$
\begin{aligned}
h_{e x} \int_{U}\left(|u|^{2}-1\right) \nabla \xi_{\ell} \cdot \nabla \zeta & \leq C h_{e x}\left\|\nabla \xi_{\ell}\right\|_{L^{\infty}}\|\nabla \zeta\|_{L^{2}}\left\|1-|u|^{2}\right\|_{L^{2}} \\
& \leq C \varepsilon h_{e x}\left\|A-h_{e x} \nabla^{\perp} \xi_{\ell}\right\|_{L^{2}} E_{\varepsilon}^{\frac{1}{2}}(u) \\
& \leq C \varepsilon \ell^{2}|\log \varepsilon|\left(\ell^{2}|\log \varepsilon|+\ell^{2}|\log \varepsilon|\right) \ell^{2}|\log \varepsilon| \\
& \leq C \varepsilon|\log \varepsilon|^{6 \gamma+3} \leq \frac{C}{|\log \varepsilon|} .
\end{aligned}
$$

For the fourth line of (2.15) we have

$$
\begin{aligned}
\frac{1}{2 \ell^{2}} \int_{U}\left|h-h_{e x}\right|^{2}+\frac{h_{e x}^{2}}{2} \int_{U}\left|\nabla^{\perp} \xi_{\ell}\right|^{2}+h_{e x} \int_{U} \nabla \xi_{\ell} \cdot \nabla \zeta \\
=\frac{1}{2 \ell^{2}} \int_{U}\left|h_{e x} \Delta \xi_{\ell}-h_{e x}+\Delta \zeta\right|^{2}+\frac{h_{e x}^{2}}{2} \int_{U}\left|\nabla \xi_{\ell}\right|^{2}+h_{e x} \int_{U} \nabla \xi_{\ell} \cdot \nabla \zeta \\
=\frac{h_{e x}^{2}}{2 \ell^{2}} \int\left|\Delta \xi_{\ell}-1\right|^{2}+\frac{h_{e x}^{2}}{2} \int\left|\nabla \xi_{\ell}\right|^{2}+\frac{1}{2 \ell^{2}} \int|\Delta \zeta|^{2} \\
\quad+\frac{h_{e x}}{\ell^{2}} \int\left(\Delta \xi_{\ell}-1\right) \Delta \zeta+h_{e x} \int \nabla \xi_{\ell} \cdot \nabla \zeta
\end{aligned}
$$

Multiplying $\zeta$ against (2.4) and integrating over $U$ we have

$$
0=-\frac{1}{\ell^{2}} \int_{U} \Delta \zeta\left(\Delta \xi_{\ell}-1\right)-\int_{U} \nabla \xi_{\ell} \cdot \nabla \zeta,
$$

then

$$
\begin{gathered}
\frac{1}{2 \ell^{2}} \int_{U}\left|h-h_{e x}\right|^{2}+\frac{h_{e x}^{2}}{2} \int_{U}\left|\nabla^{\perp} \xi_{\ell}\right|^{2}+h_{e x} \int_{U} \nabla \xi_{\ell} \cdot \nabla \zeta \\
=G^{0}+\frac{1}{2 \ell^{2}} \int_{U}|\Delta \zeta|^{2} .
\end{gathered}
$$

Combining (2.15), the bounds on the third line, and (2.16) yields (2.13).

We can now prove Proposition 2.5 by carefully extracting the concentration of the Ginzburg-Landau energy against the magnetic field potential $\xi_{\ell}$. Note that there are potentially an unbounded number of vortices, so we need to extract good decay on each vortex ball.

Proof of Proposition 2.5. We follow the approach in [18] for $\ell \equiv 1$. The first step is to establish the concentration in the cross term $\int \nabla^{\perp} \xi_{\ell} \cdot j(u)$. In particular we claim

$$
\left|\int_{U}\left(\nabla u,-i h_{e x} \nabla^{\perp} \xi_{\ell} u\right)-2 \pi h_{e x} \sum_{i \in I} d_{i} \xi_{\ell}\left(a_{i}\right)\right| \leq \frac{C}{|\log \varepsilon|} .
$$


where $a_{j}$ is the center of the vortex ball $B_{r_{i}}$ and $I$ is the vortex ball collection.

Step 1. Since $E_{\varepsilon}(u) \leq C \ell^{4}|\log \varepsilon|^{2} \leq C|\log \varepsilon|^{4 \gamma+2}$ and $h_{e x}=C \ell^{2}|\log \varepsilon|=$ $C|\log \varepsilon|^{2 \gamma+1}$ then by Proposition 2.3 we have balls $\left\{B_{r_{i}}\right\}_{i \in I}$ such that

$$
\left\{|u|<\frac{3}{4}\right\} \subset \cup_{i \in I} B_{r_{i}} \quad \text { card } I \leq C|\log \varepsilon|^{4 \gamma+2} \quad r_{i} \leq \frac{C}{|\log \varepsilon|^{10 \gamma+6}}
$$

if $\overline{B_{r_{i}}} \subset U$ and $d_{i}=\operatorname{deg}\left(u, \partial B_{r_{i}}\right)$ then

$$
\int_{B_{r_{i}}} e_{\varepsilon}(u) d x \geq \pi\left|d_{i}\right|(|\log \varepsilon|-O(\log |\log \varepsilon|)) .
$$

where we chose $\alpha=10 \gamma+6$ in Proposition 2.3. Therefore,

$$
\begin{aligned}
\left|\int_{\cup_{I} B_{r_{i}}}\left(\nabla u,-i h_{e x} \nabla^{\perp} \xi_{\ell} u\right)\right| & \leq(\operatorname{card} I) h_{e x}\|\nabla u\|_{L^{2}} \max _{i \in I} r_{i} \\
& \leq C|\log \varepsilon|^{8 \gamma+4-\alpha} \leq \frac{C}{|\log \varepsilon|}
\end{aligned}
$$

Setting $\widetilde{U}=U \backslash \cup_{i \in I} B_{r_{i}}$ then for $v=\frac{u}{|u|}$ we have

$$
\int_{\widetilde{U}}\left(\nabla u,-i h_{e x} \nabla^{\perp} \xi_{\ell} u\right)=h_{e x} \int_{\widetilde{U}} \nabla \xi_{\ell} \times j(v)+h_{e x} \int_{\widetilde{U}} \nabla \xi_{\ell} \times(j(u)-j(v)) .
$$

The second term is small, using (2.8) and (2.11), since

$$
\begin{aligned}
h_{e x} \int_{\widetilde{U}} \nabla \xi_{\ell} \times(j(u)-j(v)) & \leq h_{e x}\left\|\nabla^{\perp} \xi_{\ell}\right\|_{L^{\infty}} \int_{\widetilde{U}}\left|j(u)-\frac{j(u)}{|u|^{2}}\right| \\
& \leq C h_{e x} \int_{\widetilde{U}} \frac{|j(u)|}{|u|} \frac{|u|^{2}-1 \mid}{|u|} \leq C \varepsilon h_{e x} E_{\varepsilon}(u) \\
& \leq C \varepsilon|\log \varepsilon|^{6 \gamma+3}=\frac{C}{|\log \varepsilon|}
\end{aligned}
$$

where we used $|u| \geq \frac{1}{2}$ in $\widetilde{U}$ in the second line. Therefore,

$$
\left|\int_{\widetilde{U}}\left(\nabla u,-i h_{e x} \nabla^{\perp} \xi_{\ell} u\right)-h_{e x} \int_{\widetilde{U}} \nabla \xi_{\ell} \times j(v)\right| \leq \frac{C}{|\log \varepsilon|} .
$$

Next for $J=\left\{i\right.$ such that $\left.\overline{B_{r_{i}}} \subset U\right\}$ we claim we can extract the following bound:

$$
\left|h_{e x} \int_{B_{r_{i}}} \nabla \xi_{\ell} \times j(v)-2 \pi h_{e x} d_{i} \xi_{\ell}\left(a_{i}\right)\right| \leq \frac{C}{|\log \varepsilon|^{4 \gamma+3}}
$$


For $\Omega_{i}=B_{r_{i}} \cap\left\{x \in U\right.$ such that $\left.|u| \leq \frac{1}{2}\right\}$ then $\Omega_{i} \cap \partial B_{r_{i}}=\varnothing$. Since $|u| \geq \frac{1}{2}$ in $\Omega_{i}$ then by Stokes' theorem,

$$
\begin{aligned}
& h_{e x}\left|\int_{\partial B_{r_{i}}}\left(\xi_{\ell}-\xi_{\ell}\left(a_{i}\right)\right) j(v) \cdot \tau-\int_{\partial U_{i}}\left(\xi_{\ell}-\xi_{\ell}\left(a_{i}\right)\right) j(v) \cdot \tau\right| \\
& \quad=h_{e x}\left|\int_{B_{i} \backslash U_{i}} \nabla \xi_{\ell} \times j(u)\right| \leq C h_{e x}\left\|\nabla \xi_{\ell}\right\|_{L^{\infty}}\|\nabla u\|_{L^{2}} r_{i} \\
& \quad \leq C|\log \varepsilon|^{4 \gamma+2-\alpha} \leq \frac{C}{|\log \varepsilon|^{4 \gamma+3}},
\end{aligned}
$$

thus

$$
\begin{array}{r}
h_{e x}\left|\int_{\partial B_{r_{i}}}\left(\xi_{\ell}-\xi_{\ell}\left(a_{i}\right)\right) j(v) \cdot \tau-\int_{\partial \Omega_{i}}\left(\xi_{\ell}-\xi_{\ell}\left(a_{i}\right)\right) j(v) \cdot \tau\right| \\
\leq \frac{C}{|\log \varepsilon|^{4 \gamma+3}} .
\end{array}
$$

On the other hand since $|u|=\frac{1}{2}$ on $\partial \Omega_{i}$ we find

$$
\begin{aligned}
h_{e x} \mid & \int_{\partial \Omega_{i}}\left(\xi_{\ell}-\xi_{\ell}\left(a_{i}\right)\right) j(v) \cdot \tau \mid \\
& =h_{e x}\left|\int_{\partial \Omega_{i}}\left(\xi_{\ell}-\xi_{\ell}\left(a_{i}\right)\right) \frac{j(u)}{|u|^{2}} \cdot \tau\right|=4 h_{e x}\left|\int_{\partial \Omega_{i}}\left(\xi_{\ell}-\xi_{\ell}\left(a_{i}\right)\right) j(u) \cdot \tau\right| \\
& =4 h_{e x}\left|\int_{\Omega_{i}} \operatorname{curl}\left[\left(\xi_{\ell}-\xi_{\ell}\left(a_{i}\right)\right) j(u)\right]\right| \\
& \leq 4 h_{e x}\left|\int_{\Omega_{i}} \nabla \xi_{\ell} \times j(u)\right|+8 h_{e x}\left|\int_{\Omega_{i}}\left(\xi_{\ell}-\xi_{\ell}\left(a_{i}\right)\right) J(u)\right| \\
& \leq C h_{e x}\left\|\nabla \xi_{\ell}\right\|_{L^{\infty}}\|\nabla u\|_{L^{2}} r_{i}+C h_{e x}\left\|\nabla \xi_{\ell}\right\|_{L^{\infty}}\|\nabla u\|_{L^{2}}^{2} r_{i} \\
& \leq C|\log \varepsilon|^{4 \gamma+2-\alpha}+C|\log \varepsilon|^{6 \gamma+3-\alpha} \leq \frac{C}{|\log \varepsilon|^{4 \gamma+3}}
\end{aligned}
$$

and consequently, since card $I \leq C|\log \varepsilon|^{4 \gamma+2}$ and $\int_{\partial B_{r_{i}}} \xi_{\ell}\left(a_{i}\right) j(v) \cdot \tau=2 \pi d_{i}$, then

$$
\sum_{i \in J}\left|h_{e x} \int_{\partial B_{r_{i}}} \xi_{\ell} j(v) \cdot \tau-h_{e x} \int_{\partial B_{r_{i}}} \xi_{\ell}\left(a_{i}\right) j(v) \cdot \tau\right| \leq \frac{C}{|\log \varepsilon|}
$$


Finally, for the balls that intersect $\partial U, I \backslash J$. Since $\xi_{\ell}=0$ on $\partial U$ then for $\Omega_{i}=B_{i} \cap\left\{x \in U\right.$ such that $\left.|u| \leq \frac{1}{2}\right\}$ we follow the above argument and see

$$
\begin{aligned}
\left|h_{e x} \int_{\partial B_{r_{i}} \cap U} \xi_{\ell j} j(v) \cdot \tau\right| & \leq\left|h_{e x} \int_{\partial\left(\Omega_{i} \cap U\right)} \xi_{\ell} j(v) \cdot \tau\right|+\frac{C}{|\log \varepsilon|^{4 \gamma+3}} \\
& \leq 4 h_{e x}\left|\int_{\Omega_{i} \cap U} \nabla \xi_{\ell} \times j(v)+2 \xi_{\ell} J(v)\right|+\frac{C}{|\log \varepsilon|^{4 \gamma+3}} \\
& \leq \frac{C}{|\log \varepsilon|^{4 \gamma+3}} .
\end{aligned}
$$

Combining this estimate along with $\operatorname{card} I \leq C|\log \varepsilon|^{4 \gamma+2}$ and (2.21) yields estimate (2.17).

Step 2. We bound $\int_{U}\left|\nabla u-i \nabla^{\perp} \zeta u\right|^{2} \geq \int_{\cup_{i \in I} B_{r_{i}}}|\nabla u|^{2}-\frac{C}{|\log \varepsilon|}$. In particular

$$
\begin{aligned}
\int_{U}\left|\nabla u-i \nabla^{\perp} \zeta u\right|^{2} & \geq \int_{\cup_{i \in I} B_{r_{i}}}\left|\nabla u-i \nabla^{\perp} \zeta u\right|^{2} \\
& =\int_{\cup_{i \in I} B_{r_{i}}}|\nabla u|^{2}-2 \nabla^{\perp} \zeta \cdot j(u)+|\nabla \zeta|^{2}|u|^{2} .
\end{aligned}
$$

¿From (2.12) we see $\|A\|_{L^{\infty}(U)} \leq C\|A\|_{H^{2}(U)} \leq C|\log \varepsilon|^{3 \gamma+2}$, thus

$$
\begin{aligned}
\left|\int_{\cup_{i \in I} B_{r_{i}}} \nabla^{\perp} \zeta \cdot j(u)\right| & \leq(\operatorname{card} I)\left\|A-h_{e x} \nabla^{\perp} \xi_{\ell}\right\|_{L^{\infty}}\|\nabla u\|_{L^{2}} \max _{i \in I} r_{i} \\
& \leq C|\log \varepsilon|^{4 \gamma+2}\left(|\log \varepsilon|^{3 \gamma+2}+|\log \varepsilon|^{2 \gamma+1}\right)|\log \varepsilon|^{2 \gamma+1}|\log \varepsilon|^{-\alpha} \\
& \leq C|\log \varepsilon|^{9 \gamma+5-\alpha} \leq \frac{C}{|\log \varepsilon|},
\end{aligned}
$$

and so

$$
\int_{U}\left|\nabla u-i \nabla^{\perp} \zeta u\right|^{2} \geq \int_{\cup_{i \in I} B_{r_{i}}}|\nabla u|^{2}
$$

Combining (2.13) with (2.17) and (2.22) yields (2.5).

We are finally in the position to establish the

Proof of Proposition 2.1. The first part of the proof establishes that a minimizing sequence must be in the Meissner state when $h_{e x}<\frac{\ell^{2}|\log \varepsilon|}{2}$. 
Step 1. From Proposition 2.5 and the minimality of $(u, A)$

$$
\begin{aligned}
G^{0} & \geq G_{\varepsilon}(u, A) \\
& \geq \sum_{i \in I} \int_{B_{r_{i}}} e_{\varepsilon}(u) d x+\frac{1}{2 \ell^{2}} \int_{U}|\Delta \zeta|^{2}+G^{0}+2 \pi h_{e x} \sum_{i \in I} d_{i} \xi_{\ell}\left(a_{i}\right)-\frac{C}{|\log \varepsilon|} .
\end{aligned}
$$

Therefore, since $\xi_{\ell} \leq 0$, we use (2.7) and lower bound (4) in Proposition 2.3 to get

$$
\begin{aligned}
\pi \sum_{i \in I}\left|d_{i}\right|(|\log \varepsilon|+O(\log |\log \varepsilon|)) & \leq 2 \pi h_{e x} \sum_{i \in I} d_{i}\left|\xi_{\ell}\left(a_{i}\right)\right| \\
& \leq 2 \pi h_{e x}\left(\sum_{i \in I}\left|d_{i}\right|\right) \max \left|\xi_{\ell}\right| \\
& \leq\left(\sum_{i \in I}\left|d_{i}\right|\right) 2 \pi \frac{h_{e x}}{\ell^{2}}\left(1-o_{\ell}\right) .
\end{aligned}
$$

So if $\sum_{i \in I}\left|d_{i}\right| \neq 0$ then

$$
h_{e x} \geq \frac{\ell^{2}}{2}(|\log \varepsilon|+O(\log |\log \varepsilon|)) .
$$

Hence, for $h_{e x}<\frac{\ell^{2}}{2}|\log \varepsilon|$ then either $\operatorname{deg}\left(u, \partial B_{r_{i}}\right)=0$ or $B_{r_{i}} \cap U \neq \varnothing$. It is straightforward to show from this point that $|u| \geq \frac{3}{4}$ in $U$, see $[3,4]$.

Step 2. We now complete the proof of the critical field strength. In particular we show that if $h_{e x}>\frac{\ell^{2}|\log \varepsilon|}{2}$ then there must be a vortex. We prove this by contradiction. Let $\left(u_{\varepsilon}, A_{\varepsilon}\right)$ be a minimizing sequence with $\sum_{j \in J}\left|d_{j}\right|=0$ then we claim $G_{\varepsilon}(u, A) \geq G_{\varepsilon}\left(1, h_{e x} \nabla^{\perp} \xi_{\ell}\right)-\frac{C}{|\log \varepsilon|}$.

In order to get better bounds on $\nabla^{\perp} \zeta=A-h_{e x} \nabla^{\perp} \xi_{\ell}$, we replace lower bound (2.22) with

$$
\frac{1}{2} \int_{U}\left|\nabla u-i \nabla^{\perp} \zeta u\right|^{2} \geq \frac{1}{2} \int_{U}|\nabla u|^{2}-2 j(u) \cdot \nabla^{\perp} \zeta+|\nabla \zeta|^{2}-\frac{C}{|\log \varepsilon|},
$$

where we used the argument for the estimate of the third line of (2.15) in the proof of Lemma 2.8. By (2.8) and (2.12) we see that $\zeta$ is continuous. Since there are no nontrivial-degree vortex balls, then by an argument identical to the proof of (2.17) we have the lower bound

$$
G_{\varepsilon}(u, A) \geq E_{\varepsilon}(u)+G^{0}+\frac{1}{2} \int_{U}|\nabla \zeta|^{2}+\frac{1}{\ell^{2}}|\Delta \zeta|^{2}-\frac{C}{|\log \varepsilon|} .
$$

Since $(u, A)$ is an energy minimizer, $G^{0} \geq G_{\varepsilon}(u, A)$, and so $E_{\varepsilon}(u)+\frac{1}{2} \int_{U}|\nabla \zeta|^{2} \leq$ $\frac{C}{|\log \varepsilon|}$. Even more so, the boundary condition $\zeta=0$ implies $\zeta \rightarrow 0$ and $E_{\varepsilon}(u) \rightarrow 0$ 
as $\varepsilon \rightarrow 0$. We see that

$$
G_{\varepsilon}(u, A) \geq G^{0}-\frac{C}{|\log \varepsilon|},
$$

when $\sum_{j \in J}\left|d_{j}\right|=0$.

To prove that $G_{\varepsilon}(u, A)$ is no longer the Meissner state, we construct a sequence of functions $\left(u_{\varepsilon}, A_{\varepsilon}\right)$ which have lower energy than the Meissner energy when $h_{e x}>\frac{\ell^{2}|\log \varepsilon|}{2}$. Set $A_{\varepsilon}=h_{e x} \nabla^{\perp} \xi_{\ell}+\nabla^{\perp} \zeta$, where $\xi_{\ell}$ is defined in (2.4) and

$$
-\frac{1}{\ell^{2}} \Delta^{2} \zeta+\Delta \zeta=2 \pi \delta_{a} \text { in } U \quad \Delta \zeta=\xi_{\ell}=0 \text { on } \partial U
$$

To define $u_{\varepsilon}=\rho_{\varepsilon} e^{i \varphi_{\varepsilon}}$ we set $\nabla \varphi_{\varepsilon}=A_{\varepsilon}+\frac{1}{\ell^{2}} \nabla^{\perp} \operatorname{curl} A_{\varepsilon}$ and

$$
\rho_{\varepsilon}= \begin{cases}0 & |x-a| \leq \frac{\varepsilon}{2} \\ 1 & |x-a| \geq \varepsilon\end{cases}
$$

Then for any $B_{R} \supset\{a\}, \int_{\partial B_{R}} \partial_{\tau} \varphi_{\varepsilon}=\int_{B_{R}} h_{\varepsilon}-\frac{1}{\ell^{2}} \Delta h_{\varepsilon}=2 \pi$, which correctly quantizes the phase. A straightforward calculation shows that $E_{\varepsilon}\left(u_{\varepsilon}\right) \leq$ $\pi \log \frac{\operatorname{diam} U}{\varepsilon}+C \leq \pi|\log \varepsilon|+C$, where $C$ is a fixed constant. The arguments in Section 4 contain more refined upper bound calculations; however, they are similar in spirit.

Following Step 1 of the proof of Proposition 2.5 yields

$$
\begin{aligned}
& \frac{1}{2} \int_{U} A_{\varepsilon}^{2}\left|u_{\varepsilon}\right|^{2}+\frac{1}{\ell^{2}}\left|\operatorname{curl} A_{\varepsilon}-h_{e x}\right|^{2} \\
& \leq \frac{h_{e x}^{2}}{2} \int_{U}\left|\nabla \xi_{\ell}\right|^{2}+\frac{1}{\ell^{2}}\left|\Delta \xi_{\ell}-h_{e x}\right|^{2}+\frac{1}{2} \int_{U}|\nabla \zeta|^{2}+\frac{1}{\ell^{2}}|\Delta \zeta|^{2}+\frac{C}{|\log \varepsilon|} .
\end{aligned}
$$

Again we decompose $\left\|\nabla u-i \nabla^{\perp} \zeta u\right\|_{L^{2}(U)}^{2}=\|\nabla u\|_{L^{2}(U)}^{2}-\int j(u) \cdot \nabla^{\perp} \zeta+\left\|\nabla^{\perp} \zeta u\right\|_{L^{2}(U)}^{2}$, and a similar calculation as in Step 1 shows

$$
\begin{aligned}
G_{\varepsilon}\left(u_{\varepsilon}, A_{\varepsilon}\right) \leq & E_{\varepsilon}\left(u_{\varepsilon}\right)-\int_{U} j\left(u_{\varepsilon}\right) \cdot \nabla^{\perp}\left(h_{e x} \xi_{\ell}+\zeta\right)+G^{0} \\
& +\frac{1}{2} \int_{U}|\nabla \zeta|^{2}+\frac{1}{\ell^{2}}|\Delta \zeta|^{2}+\frac{C}{|\log \varepsilon|} \\
\leq & G^{0}+\pi|\log \varepsilon|-\frac{2 \pi h_{e x}}{\ell^{2}}\left(1-o_{\ell}(1)\right)+C \\
& +\frac{1}{2} \int_{U}|\nabla \zeta|^{2}+\frac{1}{\ell^{2}}|\Delta \zeta|^{2}+2 \pi \zeta(a),
\end{aligned}
$$

where we used (2.7) in the last inequality. Multiplying (2.24) by $\zeta$ and integrating over $U$ shows $2 \pi \zeta(a)=-\int_{U}|\nabla \zeta|^{2}+\frac{1}{\ell^{2}}|\Delta \zeta|^{2}<0$, hence $\frac{1}{2} \int_{U}|\nabla \zeta|^{2}+$ 
$\frac{1}{\ell^{2}}|\Delta \zeta|^{2}+2 \pi \zeta(a)<0$. Therefore,

$$
G_{\varepsilon}\left(u_{\varepsilon}, A_{\varepsilon}\right) \leq G^{0}+\pi|\log \varepsilon|-\frac{2 \pi h_{e x}}{\ell^{2}}\left(1-o_{\ell}(1)\right)+C .
$$

Since $h_{e x}>\frac{\ell^{2}|\log \varepsilon|}{2}$, then there exists $\delta>0$, bounded away from zero, such that $\pi|\log \varepsilon|-\frac{2 \pi h_{e x}}{\ell^{2}}\left(1-o_{\ell}(1)\right)+C<C-\delta|\log \varepsilon|<0$ for $\varepsilon$ small enough, thus

$$
G_{\varepsilon}\left(u_{\varepsilon}, A_{\varepsilon}\right) \leq G^{0}+\delta|\log \varepsilon| .
$$

Therefore, a vortex-less configuration cannot be minimizing in the $h_{e x}>\frac{\ell^{2}|\log \varepsilon|}{2}$ regime.

Remark 2.9. For values of $h_{e x}$ well above the critical field, we expect the minimizers to be similar to the functions constructed in the proof of (4.2) in Section 4 .

Remark 2.10. The proof of the critical field for $\ell_{0} \in[0,+\infty)$ proceeds in the same way as for the proof of Proposition 2.1 and can be done by a suitable modification of the method in [18]. Since we handled the more difficult case $\ell \rightarrow+\infty$ such that $\ell_{\varepsilon}=|\log \varepsilon|^{\gamma}$ for some $\gamma \in \mathbb{R}^{+}$, we leave out the proof for the case $\ell_{\varepsilon} \rightarrow \ell_{0} \in[0,+\infty)$.

\section{Obstacle PRoblem for SMALl AND BOUNDED DOMAINS}

In this section, we study the functional (1.2) where $\ell_{\varepsilon} \rightarrow \ell_{0} \in[0, \infty)$, i.e. for shrinking or bounded domains $U_{\ell}$ in the critical scaling of energy and magnetic field.

The following result is a generalization of Theorem 1.3 in [9] (where it is proved for $\ell=1$ ). Closely related results in the context of the Chern-SimonsHiggs energy were shown by the authors in [11, Theorem 1.3] and [12, Theorem 3]. We state the theorem in its gauge-invariant form.

Theorem 3.1. Let $\left(u_{\varepsilon}, A_{\varepsilon}\right)$ be a sequence with $G_{\varepsilon}\left(u_{\varepsilon}, A_{\varepsilon}\right) \leq K|\log \varepsilon|^{2}$ and assume that $h_{e x}$ satisfies $\frac{h_{e x}}{|\log \varepsilon|} \rightarrow H$ for some $H \geq 0$ and $\ell_{\varepsilon} \rightarrow \ell_{0} \in[0, \infty)$. Define the following rescaled quantities:

$$
\begin{array}{r}
a_{\varepsilon}:=\frac{1}{|\log \varepsilon|} A_{\varepsilon} \\
v_{\varepsilon}:=\frac{1}{|\log \varepsilon|}\left(i u_{\varepsilon}, \nabla u_{\varepsilon}\right) \\
w_{\varepsilon}:=v_{\varepsilon}-\left|u_{\varepsilon}\right|^{2} a_{\varepsilon}
\end{array}
$$

Then curl $a_{\varepsilon}$ is weakly compact in $L^{2}(U)$, and $w_{\varepsilon}$ is weakly compact in $L^{p}$ for $1 \leq p<2$. Furthermore, $\frac{w_{\varepsilon}}{\left|u_{\varepsilon}\right|}$ converges weakly in $L^{2}$ if and only if $w_{\varepsilon}$ converges weakly, and the weak limits are equal. 
Any weak limit of $\left(w_{\varepsilon}, \operatorname{curl} a_{\varepsilon}\right)$ can be expressed in the form $(v-a$, curl $a)$ for some $(v, a) \in L^{2}\left(U ; \mathbb{R}^{2}\right) \times H^{1}\left(U ; \mathbb{R}^{2}\right)$ such that curl $v$ is a Radon measure. In addition, we have the following $\Gamma-\lim$ inf inequality:

$$
\liminf _{\varepsilon \rightarrow 0} \frac{1}{|\log \varepsilon|^{2}} G_{\varepsilon}\left(u_{\varepsilon}, A_{\varepsilon}\right) \geq G(v, a)
$$

where the limit functional $G$ is given by

$$
\begin{aligned}
& G(v, a):= \\
& \begin{cases}\frac{1}{2} \int_{U}|v-a|^{2}+\frac{1}{\ell_{0}^{2}}|\operatorname{curl} a-H|^{2}+\frac{1}{2}\|\operatorname{curl} v\|_{\mathscr{M}} & \text { if } \ell_{0}>0 \\
\frac{1}{2} \int_{U}|v-a|^{2}+\frac{1}{2}\|\operatorname{curl} v\|_{\mathscr{M}} & \text { if } \ell_{0}=0 \text { and } \operatorname{curl} a=H \\
+\infty & \text { else. }\end{cases}
\end{aligned}
$$

Conversely, for every $(v, a) \in L^{2}\left(U ; \mathbb{R}^{2}\right) \times H^{1}\left(U ; \mathbb{R}^{2}\right)$ such that curl $v$ is a Radon measure there exists approximating sequences $\left(\tilde{u}_{\varepsilon}, \tilde{A}_{\varepsilon}\right)$ such that the convergences above hold, and such that

$$
\lim _{\varepsilon \rightarrow 0} \frac{1}{|\log \varepsilon|^{2}} G_{\varepsilon}\left(\tilde{u}_{\varepsilon}, \tilde{A}_{\varepsilon}\right)=G(v, a) .
$$

Proof. It suffices to check the theorem for sequences $\left(u_{\varepsilon}, A_{\varepsilon}\right)$ that satisfy the Coulomb gauge condition $\operatorname{div} A_{\varepsilon}=0$ in $U, A \cdot \nu=0$ on $\partial U$, since $G\left(u_{\varepsilon}, A_{\varepsilon}\right)=$ $G\left(u_{\varepsilon} e^{i \chi}, A_{\varepsilon}+\nabla \chi\right)$ and the quantities $w_{\varepsilon}$ and curl $a_{\varepsilon}$ are invariant under this gauge transformation. The limit functional $G(v, a)$ also has the gauge invariance $G(v+\nabla \chi, a+\nabla \chi)=G(v, a)$.

¿From the energy bound $G_{\varepsilon}\left(u_{\varepsilon}, A_{\varepsilon}\right) \leq K|\log \varepsilon|^{2}$ we infer that

$$
\int_{U}\left|\operatorname{curl} a_{\varepsilon}-H\right|^{2} \leq 2 K \ell_{\varepsilon}^{2} \leq C
$$

since $\ell_{\varepsilon}$ is bounded, and together with $\operatorname{div} a_{\varepsilon}=0$ this implies $\left\|a_{\varepsilon}\right\|_{H^{1}(U)} \leq C$ and via Sobolev embedding $\left\|A_{\varepsilon}\right\|_{L^{p}(U)} \leq C_{p}|\log \varepsilon|$ for $p \geq 1$.

We can now establish that the BBH energy $E_{\varepsilon}\left(u_{\varepsilon}\right)$ is bounded, using the following decomposition:

$$
\left|\nabla_{A} u\right|^{2}=|\nabla u|^{2}-2 j(u) \cdot A+|u|^{2}|A|^{2}
$$

which implies that

$$
E_{\varepsilon}\left(u_{\varepsilon}\right) \leq G_{\varepsilon}\left(u_{\varepsilon}, A_{\varepsilon}\right)+\int_{U}\left|j\left(u_{\varepsilon}\right) \cdot A_{\varepsilon}\right| .
$$


As in [9], we can estimate the cross term via

$$
\begin{aligned}
\left|j\left(u_{\varepsilon}\right) \cdot A_{\varepsilon}\right| & \leq \frac{1}{4}\left|\nabla u_{\varepsilon}\right|^{2}+\left|u_{\varepsilon}\right|^{2}\left|A_{\varepsilon}\right|^{2} \\
& \leq \frac{1}{4}\left|\nabla u_{\varepsilon}\right|^{2}+\left(\left|u_{\varepsilon}\right|^{2}-1\right)\left|A_{\varepsilon}\right|^{2}+\left|A_{\varepsilon}\right|^{2} \\
& \leq \frac{1}{4}\left|\nabla u_{\varepsilon}\right|^{2}+\frac{1}{8 \varepsilon^{2}}\left(1-\left|u_{\varepsilon}\right|^{2}\right)^{2}+2 \varepsilon^{2}\left|A_{\varepsilon}\right|^{4}+\left|A_{\varepsilon}\right|^{2} \\
& \leq \frac{1}{2} E_{\varepsilon}\left(u_{\varepsilon}\right)+C \varepsilon^{2}|\log \varepsilon|^{4}+C|\log \varepsilon|^{2}
\end{aligned}
$$

and it follows that $E_{\varepsilon}\left(u_{\varepsilon}\right) \leq C|\log \varepsilon|^{2}$. We are therefore able to use the compactness results of [9] that show compactness for $v_{\varepsilon}$ and the estimate

$$
\liminf _{\varepsilon \rightarrow 0} E_{\varepsilon}\left(u_{\varepsilon}\right) \geq \frac{1}{2} \int_{U}|v|^{2}+\frac{1}{2}\|\operatorname{curl} v\|_{\mathscr{M}} .
$$

It is then not difficult to show the lower bound for the full energy using the same decomposition as above and the weak convergence of $a_{\varepsilon}$ implied by the bounds.

The $\Gamma$-limsup property (3.2) can be shown as follows: Given a limit $(v, a)$ with $\operatorname{div} a=0$, we set $\tilde{A}_{\varepsilon}=a|\log \varepsilon|$ and construct $\tilde{u}_{\varepsilon}$ as in Section 7 of [9]. It is then easy to see that the claimed convergence holds, using the $\Gamma$-convergence result for $E_{\varepsilon}$ from [9] and the same decomposition as above.

Remark 3.2. Note that compactness for $v_{\varepsilon}$ only holds due to our choice of gauge. The representative $\tilde{u}_{\varepsilon}=u_{\varepsilon} e^{i \chi_{\varepsilon}}$ corresponds to $\tilde{v}_{\varepsilon}=v_{\varepsilon}+\frac{1}{|\log \varepsilon|} \nabla \chi_{\varepsilon}$, and so $v_{\varepsilon}$ and $\tilde{v}_{\varepsilon}$ need not have the same compactness properties. The limit functional $G(v, a)$ also has the gauge invariance $G(v+\nabla \chi, a+\nabla \chi)=G(v, a)$. If $\ell_{\varepsilon} \rightarrow \infty$, the compactness argument for $a_{\varepsilon}$ fails, since we only know that $\int_{U}\left|\operatorname{curl} a_{\varepsilon}-H\right|^{2} \leq K \ell_{\varepsilon}^{2}$, so this sequence need not be bounded. The example given in [12, Theorem 5], which also holds for (1.2), shows that also $v_{\varepsilon}$ need not be compact in this case, even if $\operatorname{div} A_{\varepsilon}=0$. In fact we construct a sequence of $\left(v_{\varepsilon}, a_{\varepsilon}\right)$ with bounded energy but $\left\|v_{\varepsilon}\right\|_{L^{2}(U)} \gtrsim \log \ell_{\varepsilon} \rightarrow+\infty$ by constructing a set of vortices that concentrate about a single point. Therefore, the energy splitting approach of [9] is insufficient to treat the case of large domains.

As in [17], we can characterize the minimizers of the limit functional. We obtain, following the presentation of [9]:

Theorem 3.3. If $\left(v_{0}, a_{0}\right)$ is a minimizer of $G$ and $\ell_{0}>0$, then $z_{0}=\ell_{0}^{-2}\left(\operatorname{curl} a_{0}-\right.$ $H)$ is the unique minimizer of the following obstacle problem: Minimize

$$
F_{\ell_{0}, H}(z)=\frac{1}{2} \int_{U}|\nabla z|^{2}+\ell_{0}^{2} z^{2}+2 z H
$$


in the admissible class

$$
\mathscr{K}=\left\{z \in H_{0}^{1}(U): z \geq-\frac{1}{2} \text { a.e. in } U\right\} .
$$

The limit $\left(v_{0}, a_{0}\right)$ satisfies the following additional properties:

$$
\begin{aligned}
\ell_{0}^{-2} \operatorname{curl}\left(\operatorname{curl} a_{0}-H\right)+a_{0} & =v_{0} \quad \text { in } U \\
\operatorname{curl} a_{0}-H & =0 \quad \text { on } \partial U \\
-\frac{1}{2} & \leq z_{0} \leq 0 \\
\operatorname{curl} v_{0} & \geq 0 \\
\operatorname{spt}\left(\operatorname{curl} v_{0}\right) & \subset\left\{z_{0}=-\frac{1}{2}\right\} .
\end{aligned}
$$

In the case where $\ell_{0}=0$, we have curl $a_{0} \equiv H$ and obtain a slightly different obstacle problem: Let $y_{0}$ be the solution of $-\Delta y_{0}=\operatorname{curl} v_{0}-H$ with zero boundary conditions. Then $y_{0}$ is the unique minimizer of

$$
F_{0, H}(y)=\frac{1}{2} \int_{U}|\nabla y|^{2}+2 y H
$$

in the admissible class

$$
\mathscr{K}=\left\{y \in H_{0}^{1}(U): y \geq-\frac{1}{2} \text { a.e. in } U\right\} .
$$

Moreover, $\operatorname{curl} v_{0} \geq 0$, and $\operatorname{spt}\left(\operatorname{curl} v_{0}\right) \subset\left\{y_{0}=-\frac{1}{2}\right\}$.

Proof. We only prove the part for $\ell_{0}=0$; the first half can be shown by a completely straightforward insertion of $\ell^{-2}$ into the argument of [9]. Our proof of the second half also follows the structure of their argument.

For a Radon measure $\mu \in H^{-1}$, define the vector field $v^{\mu} \in L^{2}\left(U ; \mathbb{R}^{2}\right)$ by $\operatorname{curl} v^{\mu}=\mu$ and $\operatorname{div} v^{\mu}=0$. We decompose $\mu$ as $\mu^{a c}+\mu^{\text {sing }}$ into an absolutely continuous and a singular part. Setting $g(t, \mu)=G\left(v_{0}+t v_{\mu}, a\right)$, we calculate $0 \leq \lim _{t \rightarrow 0^{+}} \frac{g(t, \mu)-g(0, \mu)}{t}=\int_{U}\left(v_{0}-a_{0}, v^{\mu}\right)+\frac{1}{2} \int_{U} \operatorname{sgn}\left(\mu_{0}\right) d \mu^{a c}+\frac{1}{2}\left\|\mu^{\text {sing }}\right\|(U)$.

Integrating by parts and using the definition of $y_{0}$, we see that

$$
\int_{U}\left(v_{0}-a_{0}, v^{\mu}\right)=\int_{U} y_{0} \mu
$$

so we obtain

$$
0 \leq \int_{U}\left(y_{0}+\frac{1}{2} \operatorname{sgn}\left(\mu_{0}\right)\right) d \mu^{a c}+\int_{U}\left(y_{0}+\frac{1}{2} \operatorname{sgn}(\mu)\right) d \mu^{\text {sing }}
$$

and similarly by one-sided differentiation in the opposite direction

$$
0 \geq \int_{U}\left(y_{0}+\frac{1}{2} \operatorname{sgn}\left(\mu_{0}\right)\right) d \mu^{a c}+\int_{U}\left(y_{0}-\frac{1}{2} \operatorname{sgn}(\mu)\right) d \mu^{\text {sing }} .
$$


Together, (3.3) and (3.4) imply, due to the arbitrariness of $\mu^{a c}$ and $\mu^{\text {sing }}$, that $\left|y_{0}\right| \leq \frac{1}{2}$ everywhere and $y_{0}=-\frac{1}{2} \operatorname{sgn}\left(\mu_{0}\right)$ in spt $\mu_{0}$. It follows that for any smooth function $\varphi$ with $\varphi(z)=0$ for $z \leq 0$ and $\varphi^{\prime}(z) \geq 0$, there holds

$$
\int_{U} \varphi\left(y_{0}\right) d \mu_{0}=-\varphi\left(\frac{1}{2}\right) \mu_{0}^{-}(U)
$$

where $\mu_{0}^{-}$denotes the negative part in the Hahn decomposition of $\mu_{0}$. Since $\mu_{0}=-\Delta y_{0}+H$, we can integrate by parts and obtain

$$
\int_{U} \varphi\left(y_{0}\right) d \mu_{0}=\int_{U} \varphi^{\prime}\left(y_{0}\right)\left|\nabla y_{0}\right|^{2}+\varphi\left(y_{0}\right) H \geq 0
$$

and we conclude that $\mu_{0}^{-}(U)=0$ so $\mu_{0} \geq 0$.

To see that $y_{0}$ is a solution of the obstacle problem, we take any $y \in \mathscr{K}$ and compare using $|v|^{2}-|w|^{2} \geq 2(v-w) \cdot w$ and integration by parts

$$
F_{0, H}(y)-F_{0, H}\left(y_{0}\right) \geq \int_{U} \nabla\left(y-y_{0}\right) \cdot \nabla y_{0}+\left(y-y_{0}\right) H=\int_{U}\left(y-y_{0}\right) d \mu_{0} .
$$

Now $y_{0}=-\frac{1}{2}$ on $\operatorname{spt}\left(\mu_{0}\right)$, so $\left(y-y_{0}\right) \geq 0$ on $\operatorname{spt}\left(\mu_{0}\right)$ for all $y \in \mathscr{K}$. It follows that $y_{0}$ is a minimizer of the obstacle problem. Standard theory [6] can now be used to show uniqueness of $y_{0}$.

Remark 3.4. We believe the family of obstacle problems in Theorem 3.3 can be studied in the framework of Brezis-Serfaty [5], who examine the obstacle problem arising from (1.3).

Corollary 3.5. Let $\left(v_{0}, a_{0}\right)$ be a minimizer of $G(v, a)$. Then curl $v_{0}=0$ for $H<H_{1}\left(\ell_{0}\right)$ and curl $v_{0} \neq 0$ for $H>H_{1}\left(\ell_{0}\right)$, where $H_{1}\left(\ell_{0}\right)$ is given by

$$
H_{1}\left(\ell_{0}\right)=\frac{1}{2 \max _{\bar{U}}\left|y_{\ell_{0}}\right|},
$$

where $y_{\ell_{0}}$ is the solution of

$$
-\Delta y_{\ell_{0}}+\ell_{0}^{2} y_{\ell_{0}}+1=0
$$

with Dirichlet boundary conditions $y_{\ell_{0}}=0$ on $\partial U$.

Remark 3.6. We reiterate that the function $H_{1}$ in (3.5) satisfies $\frac{2 H_{1}\left(\ell_{0}\right)}{\ell_{0}^{2}} \rightarrow 1$ as $\ell_{0} \rightarrow \infty$.

Remark 3.7. In the case where $U=B_{1}(0)$ is a ball, the function $H_{1}$ can be written down explicitly since the solutions of $-\Delta y+\alpha y+1=0$ with Dirichlet boundary conditions are given by known special functions. Denoting by $I_{0}$ the modified Bessel function of zeroeth order, we have that

$$
H_{1}\left(\ell_{0}\right)=\frac{\ell_{0}^{2} I_{0}\left(\ell_{0}\right)}{2\left(I_{0}\left(\ell_{0}\right)-1\right)}
$$


Since $I_{0}(x) \sim \frac{e^{x}}{\sqrt{2 \pi x}}$ as $x \rightarrow \infty$ and $I_{0}(x)=1+\frac{x^{2}}{4}+O\left(x^{4}\right)$ as $x \rightarrow 0$, it is easy to see that this matches the claimed behavior at zero and infinity.

\section{UPPER BOUND FOR VORTEX LATTICES}

In this section, we construct good comparison sequences that correpond to vortex lattices and calculate their energy.

Proposition 4.1. Assume $\varepsilon<\frac{C}{\sqrt{h_{e x}}}$ and $\varepsilon \rightarrow 0$. There exists a sequence of functions $\left(u_{\varepsilon}, A_{\varepsilon}\right)$ such that the Ginzburg-Landau energy satisfies

$$
G_{\varepsilon}\left(u_{\varepsilon}, A_{\varepsilon}\right) \leq h_{e x} \frac{|U|}{2}\left(\log \frac{1}{\sqrt{h_{e x}} \varepsilon}+C\right) .
$$

If $h_{e x}-\frac{\ell^{2}|\log \varepsilon|}{2}=S \gg 1$ and $h_{e x} \leq \frac{1}{\varepsilon^{2}}$ then there exists a sequence of functions with Ginzburg-Landau energy

$$
G_{\varepsilon}\left(u_{\varepsilon}, A_{\varepsilon}\right) \leq \frac{|U|}{2}\left(S(|\log (\varepsilon \max (\sqrt{S}, \ell))|+C)+\frac{\ell^{2}}{4}|\log \varepsilon|^{2}\right) .
$$

Remark 4.2. If $\ell^{2} \geq K h_{e x}$ then

$$
G_{\varepsilon}(1,0) \leq h_{e x} \frac{|U|}{2 K}
$$

In particular, there is a constant $K>0$ such that our vortex lattice construction is not minimizing for $\ell^{2} \geq K h_{e x}$.

Remark 4.3. Under the assumptions for the upper bound (4.2), the trivial Meissner-like state $(u, A)=(1,0)$ has the energy

$$
\frac{|U|}{2}\left(\frac{S^{2}}{\ell^{2}}+S|\log \varepsilon|+\frac{\ell^{2}}{4}|\log \varepsilon|^{2}\right) .
$$

Since $|\log (\varepsilon \max (\ell, \sqrt{S}))| \leq|\log \varepsilon|-C$, the vortex lattice state with $O(S)$ vortices is energetically favorable compared to $(u, A)=(1,0)$. Consult Proposition 2.1 for a more detailed statement regarding the first critical field for vortex nucleation.

We now turn to the proof of Proposition 4.1. We present a novel approach to estimate the energy of a vortex lattice. As we are looking for periodic vortex lattices, it is natural to use Fourier series. On the unit cell of our lattice, we investigate solutions of

$$
-\alpha \Delta h+h=2 \pi \delta_{\varepsilon} \quad \text { in } K_{L}
$$

with homogeneous Neumann boundary conditions. Here $K_{L}=\left(-\frac{L}{2}, \frac{L}{2}\right)^{2}$ for some $L>0$. This is equivalent to looking at $L \mathbb{Z}^{2}$-periodic solutions in $\mathbb{R}^{2}$. For $\delta_{\varepsilon}$ we use the Dirac sequence

$$
\delta_{\varepsilon}(x)=\frac{1}{4 \varepsilon^{2}} \chi_{(-\varepsilon, \varepsilon)}\left(x_{1}\right) \chi_{(-\varepsilon, \varepsilon)}\left(x_{2}\right),
$$


where $\chi_{A}$ is the characteristic function of a set $A \subset \mathbb{R}$. We assume $2 \varepsilon<L$. We obtain the following results on the lattice:

Proposition 4.4. There exists a $C>0$ such that for any $L, \varepsilon$ with $\varepsilon<\frac{L}{2}$ there exists a periodic function $h$ such that $-\ell^{-2} \Delta h+h=\delta_{\varepsilon}$ and

$$
\int_{K_{L}} \ell^{-4}|\nabla h|^{2}+\ell^{-2}\left|h-h_{e x}\right|^{2} \leq 2 \pi \log \frac{1}{\max \left(\ell, L^{-1}\right) \varepsilon}+C+L^{2}\left(h_{e x}-\frac{2 \pi}{L^{2}}\right)^{2} .
$$

Proof. We calculate the energy

$$
\int_{K_{L}} \alpha^{2}|\nabla h|^{2}+\ell^{-2}\left|h-h_{e x}\right|^{2}
$$

It will become apparent later that we should use $\alpha=\ell^{-2}$.

We use double Fourier series as follows. For $f \in L^{2}\left(K_{L}\right)$ and $k \in \mathbb{Z}^{2}$, set

$$
a_{k}=\frac{1}{L^{2}} \int_{K_{L}} f(x) e^{-i \gamma k \cdot x}
$$

where $\gamma=\frac{2 \pi}{L}$. Then $f$ can be reconstructed as

$$
f(x)=\sum_{k \in \mathbb{Z}^{2}} a_{k} e^{i \gamma k \cdot x}
$$

By Plancherel's theorem we have

$$
\int_{K_{L}}|f|^{2}=L^{2} \sum_{k \in \mathbb{Z}^{2}}\left|a_{k}\right|^{2}
$$

It is standard that $\nabla f$ corresponds to the series $\left(i \gamma k a_{k}\right)$ and $\Delta f$ to the series $\left(-\gamma^{2}|k|^{2} a_{k}\right)$. Solving the equation (4.3) therefore corresponds to

$$
\left(\alpha \gamma^{2}|k|^{2}+1\right) a_{k}=b_{k}
$$

where $b_{k}$ are the Fourier coefficients for $\delta_{\varepsilon}$.

We calculate these coefficients. Set $k=\left(k_{1}, k_{2}\right)$. If $k_{1} k_{2} \neq 0$ then

$$
b_{k}=\frac{2 \pi}{4 \varepsilon^{2} L^{2}} \frac{4 \sin \left(\gamma k_{1} \varepsilon\right) \sin \left(\gamma k_{2} \varepsilon\right)}{\gamma^{2} k_{1} k_{2}}
$$

In other cases we have

$$
b_{(m, 0)}=b_{(0, m)}=\frac{2 \pi}{4 \varepsilon^{2} L^{2}} \frac{4 \varepsilon \sin (\gamma m \varepsilon)}{\gamma m}
$$

and finally $b_{0}=\frac{2 \pi}{L^{2}}$.

To simplify notation we write this using $\operatorname{sinc}(x)$, the continuous continuation of $\frac{\sin x}{x}$, which yields

$$
b_{k}=\frac{2 \pi}{L^{2}} \operatorname{sinc}\left(\gamma k_{1} \varepsilon\right) \operatorname{sinc}\left(\gamma k_{2} \varepsilon\right) .
$$


Since $a_{0}=b_{0}=\frac{2 \pi}{L^{2}}$, we have that

$$
\int\left|h-h_{e x}\right|^{2}=L^{2} \sum_{k \neq 0}\left|a_{k}\right|^{2}+L^{2}\left(\frac{2 \pi}{L^{2}}-h_{e x}\right)^{2} .
$$

We want to calculate

$$
E=L^{2} \sum_{k \in \mathbb{Z}^{2} \backslash 0}\left(\alpha^{2} \gamma^{2}|k|^{2}+\alpha\right)\left|a_{k}\right|^{2}+L^{2}\left(\frac{2 \pi}{L^{2}}-h_{e x}\right)^{2} .
$$

Using the expressions obtained for $b_{k}$ above, it follows that we have

$$
E=\frac{4 \pi^{2}}{L^{2}} \sum_{k \in \mathbb{Z}^{2} \backslash 0} \frac{\alpha}{\alpha|\gamma|^{2}|k|^{2}+1} \operatorname{sinc}^{2}\left(\gamma \varepsilon k_{1}\right) \operatorname{sinc}^{2}\left(\gamma \varepsilon k_{2}\right)+L^{2}\left(\frac{2 \pi}{L^{2}}-h_{e x}\right)^{2} .
$$

We split up the double sum as follows. First, consider $k=\left(k_{1}, k_{2}\right)$ with $1 \leq$ $|k| \leq \frac{1}{\gamma \varepsilon}$. For these terms we estimate $\mid$ sinc $\mid \leq 1$. We label this part of the energy $E_{1}$, and so

$$
E_{1} \leq \frac{4 \pi^{2}}{L^{2}} \sum_{K_{1}} \frac{\alpha}{\alpha \gamma^{2}|k|^{2}+1}
$$

Now we compare the sum with an integral. For any decreasing function $f$, we have

$$
\sum_{1 \leq|k| \leq A} f(|k|) \leq \int_{1-\frac{1}{\sqrt{2}}}^{A+\frac{1}{\sqrt{2}}} f(r) 2 \pi r d r
$$

and so

$$
E_{1} \leq \frac{4 \pi^{2}}{L^{2}} \int_{1-c}^{\frac{1}{\gamma \varepsilon}+c} \frac{2 \pi \alpha r}{1+\alpha \gamma^{2} r^{2}} d r=\left.\frac{4 \pi^{2}}{L^{2} \gamma^{2}}\left(\frac{2 \pi}{2} \log \left(\alpha \gamma^{2} x^{2}+1\right)\right)\right|_{x=1-c} ^{x=\frac{1}{\gamma \varepsilon}+c}
$$

where $c=\frac{1}{\sqrt{2}}$. As $\frac{4 \pi^{2}}{L^{2} \gamma^{2}}=1$, we obtain

$$
E_{1} \leq \frac{2 \pi}{2} \log \frac{\frac{\alpha}{\varepsilon^{2}}+\frac{2 c \alpha \gamma}{\varepsilon}+c^{2}+1}{\alpha \gamma^{2}(1-c)^{2}+1} .
$$

We distinguish two cases. If $\alpha \gamma^{2} \leq 1$, we estimate the denominator as $\geq 1$ and obtain

$$
E_{1} \leq \frac{2 \pi}{2} \log \frac{\alpha}{\varepsilon^{2}}+C \leq 2 \pi \log \frac{\sqrt{\alpha}}{\varepsilon}+C .
$$

In the case where $\alpha \gamma^{2} \geq 1$, we estimate the denominator as $\geq C \alpha \gamma^{2}$ and obtain

$$
E_{1} \leq \frac{2 \pi}{2} \log \frac{1}{\gamma^{2} \varepsilon^{2}}+C \leq 2 \pi \log \frac{1}{\gamma \varepsilon}+C
$$

if $\alpha \gamma>\varepsilon$.

We still need to deal with the frequencies $k$ with $|k| \geq \frac{1}{\gamma \varepsilon}$. We use that $\operatorname{sinc}^{2}(x) \operatorname{sinc}^{2}(y) \leq \frac{2}{r^{2}}$, which can be seen as follows. Assume without loss of 
generality that $|x| \leq|y|$. Then $r^{2}=x^{2}+y^{2} \leq 2 y^{2}$. Estimating $|\operatorname{sinc}(x)| \leq 1$ and $|\operatorname{sinc}(y)| \leq \frac{1}{y}$, we see that $\operatorname{sinc}^{2}(x) \operatorname{sinc}^{2}(y) \leq \frac{1}{y^{2}} \leq \frac{2}{r^{2}}$ as claimed.

To calculate the energy contribution $E_{2}$ of those $k$ with $|k| \geq \frac{1}{\gamma \varepsilon}$, we again replace the sum by an integral. Using the sinc bound, we see that

$$
E_{2} \leq \frac{4 \pi^{2}}{L^{2}} \int_{\frac{1}{\gamma \varepsilon}-c}^{\infty} \frac{4 \pi \alpha r}{\left(\alpha r^{2} \gamma^{2}+1\right) \gamma^{2} r^{2} \varepsilon^{2}}
$$

We estimate this using $4 \pi^{2} L^{-2} \gamma^{-2}=1$ as

$$
E_{2} \leq C \int_{\frac{1}{\gamma \varepsilon}-c}^{\infty} \frac{1}{\gamma^{2} \varepsilon^{2} r^{3}} d r \leq \frac{C}{\gamma^{2} \varepsilon^{2}} \cdot \frac{1}{\left(\frac{1}{\gamma \varepsilon}-c\right)^{2}}
$$

and for $\frac{1}{\gamma \varepsilon}>2 c$, we obtain that $E_{2} \leq C$. The claim follows using the definitions.

Choosing $L=\frac{2 \pi}{h_{e x}}$ in (4.4) implies the following upper bound:

Corollary 4.5. If $\varepsilon<\frac{1}{2 \sqrt{h_{e x}}}$ then there exists a periodic function $h$ with period $L=\sqrt{\frac{2 \pi}{h_{e x}}}$ such that $-\ell^{-2} \Delta h+h=\delta_{\varepsilon}$ and

$$
\int_{K_{L}} \ell^{-4}|\nabla h|^{2}+\ell^{-2}\left|h-h_{e x}\right|^{2} \leq 2 \pi \log \frac{1}{\max \left(\ell, \sqrt{h_{e x}}\right) \varepsilon}+C .
$$

Furthermore, for any $L>2 \varepsilon$ there exists $h$ with $-\ell^{-2} \Delta h+h=\delta_{\varepsilon}$ and

$\int_{K_{L}} \ell^{-4}|\nabla h|^{2}+\ell^{-2}\left|h-h_{e x}\right|^{2} \leq 2 \pi \log \frac{1}{\max \left(\ell, \sqrt{h_{e x}}\right) \varepsilon}+C+L^{2} \ell^{-2}\left(\frac{2 \pi}{L^{2}}-h_{e x}\right)^{2}$.

Remark 4.6. This can be easily extended to $\varepsilon \leq \frac{C}{\sqrt{h_{e x}}}$ for any $C$ that is bounded independently of $\varepsilon, \ell$, and $h_{e x}$ by choosing $\tilde{\varepsilon}=\frac{2 \varepsilon}{C}$ and constructing with $\tilde{\varepsilon}$ instead of $\varepsilon$.

To construct a pair $(u, A)$ from $h$, we do the following. To define the modulus $\rho$, we set

$$
\rho(r)= \begin{cases}0 & r<\varepsilon \sqrt{2} \\ \frac{r-\varepsilon \sqrt{2}}{\varepsilon} & \varepsilon \sqrt{2}<r<\varepsilon(1+\sqrt{2}) . \\ 1 & r>\varepsilon(1+\sqrt{2})\end{cases}
$$

We take any $A$ with $\operatorname{curl} A=h$. Outside $B_{\varepsilon \sqrt{2}}$, we define $u$ as $\rho e^{i \varphi}$, where $\nabla \varphi-A=\alpha \nabla^{\perp} h$. This is possible since for any simple closed curve $\Gamma \subset K_{L} \backslash B_{\varepsilon \sqrt{2}}$ with $\Gamma=\partial G$ we have

$$
\int_{\Gamma} \frac{\partial \varphi}{\partial \tau}=\int_{\Gamma}\left(A \cdot \tau-\alpha \frac{\partial h}{\partial \nu}=\int_{G}(-\Delta h+h)= \begin{cases}2 \pi & \text { if } G \supset B_{\varepsilon \sqrt{2}} \\ 0 & \text { else. }\end{cases}\right.
$$


On $K_{L}$, we can therefore estimate

$$
\begin{aligned}
& \frac{1}{2} \int_{K_{L}}|(\nabla-i A) u|^{2}+\ell^{-2}\left|h-h_{e x}\right|^{2}+\frac{1}{2 \varepsilon^{2}}\left(1-\rho^{2}\right)^{2} \\
& \leq \frac{1}{2} \int_{K_{L}} \rho^{2} \ell^{-4}|\nabla h|^{2}+\ell^{-2}\left|h-h_{e x}\right|^{2}+|\nabla \rho|^{2}+\frac{1}{2 \varepsilon^{2}}\left(1-\rho^{2}\right)^{2} \\
& \leq C+\pi \log \frac{1}{\max \left(\ell, \sqrt{h_{e x}}\right) \varepsilon} .
\end{aligned}
$$

We are now in the position to establish the

Proof of Proposition 4.1. This will be done in two steps.

Step 1. We use the above construction to build an $h$ in $\mathbb{R}^{2}$ and to define a periodic $\rho_{\varepsilon}$ corresponding to the lattice. As the equivalent of (4.5) holds in all of $\mathbb{R}^{2}$, we can define $(u, A)$ in all of $\mathbb{R}^{2}$ such that (4.6) holds on every cell of the lattice. All we need to do is choose a proper origin for our lattice: for any $a \in K_{L}$ we can set $\left(u^{a}(z), A^{a}(z)\right)=(u(z-a), A(z-a))$, which has energy density $g l^{a}(z)=g l(z-a)$, where $g l(z)=\frac{1}{2}|(\nabla-i A) u|^{2}(z)+\ell^{-2} \mid \operatorname{curl} A(z)-$ $\left.h_{e x}\right|^{2}+\frac{1}{2 \varepsilon^{2}}\left(1-|u(z)|^{2}\right)^{2}$. Integrating over the unit cell, we see that

$$
\int_{K_{L}} G_{\varepsilon}\left(u_{\varepsilon}^{a}, A_{\varepsilon}^{a} ; U\right) d a=\int_{K_{L}} \int_{U} g l^{a}(z) d z d a=|U| G_{\varepsilon}\left(u, A ; K_{L}\right)
$$

The mean value theorem shows that there exists $a$ such that $G\left(u^{a}, A^{a} ; U\right) \leq$ $\frac{|U|}{\left|K_{L}\right|} G\left(u, A ; K_{L}\right)$ and since $\left|K_{L}\right|=\frac{h_{e x}}{2 \pi}$, this finishes the proof of (4.1).

Step 2. We follow the argument in Step 1; however, we choose a lattice of size $L=\sqrt{\frac{2 \pi}{S}}$, which is optimal up to logarithmic terms. Since $h_{e x} \leq \varepsilon^{-2}$ and $S \gg 1$ we have $2 \varepsilon \leq L \ll 1$ we can follow the same construction as above and obtain for the energy after choosing a suitable origin

$$
\begin{array}{r}
G\left(u_{\varepsilon}, A_{\varepsilon} ; U\right) \leq \frac{|U|}{2\left|K_{L}\right|}\left(2 \pi \log \frac{1}{\max (\sqrt{S}, \ell) \varepsilon}+C+L^{2} \ell^{-2}\left|h_{e x}-\frac{2 \pi}{L^{2}}\right|\right) \\
=\frac{|U|}{2}\left(\frac{S}{2 \pi}\left(2 \pi \log \frac{1}{\max (\sqrt{S}, \ell) \varepsilon}+C\right)+\ell^{-2}\left(\frac{1}{2} \ell^{2}|\log \varepsilon|\right)^{2}\right) \\
=\frac{|U|}{2}\left(S \log \frac{1}{\max (\sqrt{S}, \ell) \varepsilon}+C+\frac{1}{4} \ell^{2}|\log \varepsilon|^{2}\right)
\end{array}
$$

since $h_{e x}-\frac{2 \pi}{L^{2}}=h_{e x}-S=\frac{1}{2} \ell^{2}|\log \varepsilon|$. This completes the proof of (4.2). 


\section{LOWER BOUND FOR VORTEX LATTICES}

We now establish the energy lower bound for supercritical magnetic fields. These lower bounds are valid for a broad regime of length-scales $\ell$. Our method revisits the approach in [16] and makes a very careful use of the length scale versus vortex ball size that is crucial in the $\ell \rightarrow \infty$ case (and not crucial in the $\ell \rightarrow[0,+\infty)$ case $)$.

Proposition 5.1. Assume that

$$
\max \left\{1, \ell^{2}\right\}|\log \varepsilon| \ll h_{e x} \ll \frac{1}{\varepsilon^{2}},
$$

then minimizing sequence $\left\{u_{\varepsilon}, A_{\varepsilon}\right\}$ satisfies:

- when $\ell^{2} \ll \frac{1}{\varepsilon^{2}|\log \varepsilon|}$ and $\ell^{2} \leq h_{e x}$ then

$$
G_{\varepsilon}\left(u_{\varepsilon}, A_{\varepsilon}\right) \geq \frac{1}{2}|U| h_{e x} \log \frac{1}{\varepsilon \sqrt{h_{e x}}}\left(1-o_{\varepsilon}(1)\right)
$$

- when $|\log \varepsilon| \ll \ell^{2} \ll \frac{1}{\varepsilon^{2}|\log \varepsilon|}$ and $h_{e x} \leq \ell^{2}$ then

$$
G_{\varepsilon}\left(u_{\varepsilon}, A_{\varepsilon}\right) \geq \frac{1}{2}|U| h_{e x} \log \frac{1}{\varepsilon \ell}\left(1-o_{\varepsilon}(1)\right) .
$$

Remark 5.2. We note that for our supercritical fields, $h_{e x} \not \leq \ell^{2}$, due to (5.1); however, we include (5.3) since it points to our conjectured energy strength for $h_{e x}-h_{c_{1}} \gg 1$, but $h_{c_{1}} \nless h_{e x}$, see (4.2). We also note that the hypothesis $\ell^{2}|\log \varepsilon| \ll h_{e x}$ is used in (5.22).

Furthermore, we can show that $\mu_{\varepsilon}=\frac{2 \pi}{h_{e x}} \sum d^{\varepsilon} \delta_{a_{j}} \rightarrow d x$, the Lebegue measure on $\mathbb{R}^{2}$. This ensures some uniformity of vorticity in the limit, i.e. the limiting measure converges to the uniform measure and hence vorticity is spread out uniformly in the limit.

Proposition 5.3. Let $u_{\varepsilon}, A_{\varepsilon}$ be a minimizer then there exists a disjoint set of balls $\left\{B_{r_{k}}\left(a_{k}\right)\right\}_{i=1}^{N}$ with

$$
r_{k} \leq h_{e x}^{-\frac{1}{2}} \quad \text { and } \quad \sum r_{k} \leq|\Omega| h_{e x}^{\frac{1}{2}}
$$

such that $\left|u_{\varepsilon}\right| \geq \frac{1}{2}$ on $\partial B_{r_{k}}\left(a_{k}\right)$. If $d_{k}=\operatorname{deg}\left(u_{\varepsilon}, \partial B_{r_{k}}\left(a_{k}\right)\right)$ then

$$
\frac{2 \pi}{h_{e x}} \sum d_{k} \delta_{a_{k}} \overrightarrow{\mathcal{M}} d x
$$

the Lebesgue measure on $\Omega$ as $\varepsilon \rightarrow 0$.

The proof of Proposition 5.3 follows from establishing a good radius for each vortex ball where the vortex structure is well-defined, and since the argument is a straightforward adaptation of arguments of [16] and the proof of Proposition 5.1, we leave out the proof. 
To establish Proposition 5.1 we emulate the approach in Sandier-Serfaty [16]. Let $K \subset U$ be a subset of $U$. Then

$$
G_{K}=\int_{K} g_{\varepsilon}\left(u_{\varepsilon}, A_{\varepsilon}\right) d x
$$

and we set $\omega_{t}=\{x \in K: \rho(x)<t\}$. Finally, we assume we have a good energy bound on the square. We choose squares $K$ of side length $\delta=\delta(\varepsilon) \rightarrow 0$ so that the following scalings hold:

$$
V_{\varepsilon} \ll h_{e x} \delta^{2} \ll \min \left\{h_{e x}, V_{\varepsilon}^{2}\right\}
$$

where

$$
V_{\varepsilon}=\log \left(\frac{1}{\varepsilon \max \left\{\ell, \sqrt{h_{e x}}\right\}}\right) .
$$

We also assume

$$
G_{K} \leq h_{e x} \delta^{2} V_{\varepsilon}
$$

otherwise our lower bound would directly follow. Let $\Theta(t)=\int_{K \backslash \omega_{t}}|\nabla \varphi-A|^{2} d x$ where $u=\rho e^{i \varphi}$. Then following [14]

$$
\begin{aligned}
\int_{K} g_{\varepsilon}(u, A) d x= & \frac{1}{2} \int_{0}^{\infty}\left[\int_{\partial \omega_{t} \cap K}|\nabla \rho|+\frac{\left(1-t^{2}\right)^{2}}{2 \varepsilon^{2}|\nabla \rho|} d l-t^{2} \Theta^{\prime}(t)\right] d t \\
& +\frac{1}{2 \ell^{2}} \int_{K}\left|h-h_{e x}\right|^{2} d x
\end{aligned}
$$

by the co-area formula. Therefore, integration by parts on the third term yields

$$
\begin{aligned}
\int_{K} g_{\varepsilon}(u, A) d x \geq \int_{0}^{1} & {\left[\int_{\partial \omega_{t} \cap K} \frac{|\nabla \rho|}{2}+\frac{\left(1-t^{2}\right)^{2}}{4 \varepsilon^{2}|\nabla \rho|} d l\right.} \\
+t & {\left.\left[\int_{K \backslash \omega_{t}}|\nabla \varphi-A|^{2} d x+\frac{1}{2 \ell^{2}} \int_{K}\left|h-h_{e x}\right|^{2} d x\right]\right] d t . }
\end{aligned}
$$

or

$$
G_{K}=\int_{K} g_{\varepsilon}(u, A) d x \geq \int_{0}^{1} a(t)+2 t b(t) d t
$$

with

$$
\begin{aligned}
a(t) & =\int_{\partial \omega_{t} \cap K} \frac{|\nabla \rho|}{2}+\frac{\left(1-t^{2}\right)^{2}}{4 \varepsilon^{2}|\nabla \rho|} d l \\
b(t) & =\frac{1}{2} \int_{K \backslash \bar{\omega}_{t}}|\nabla \varphi-A|^{2} d x+\frac{1}{2 \ell^{2}} \int_{K}\left|h-h_{e x}\right|^{2} d x .
\end{aligned}
$$

We now use the following result of [16] to bound $\int_{0}^{1} a(t) d t$ from below. 
Lemma 5.4 (Sandier-Serfaty,[16]). There exists a constant $C$ such that

$$
\int_{0}^{1} a(t) d t \geq C \int_{\frac{\varepsilon}{\delta}}^{1} \frac{r\left(\Omega_{t}\right)\left(1-t^{2}\right)}{\varepsilon} d t
$$

where $\Omega_{t}=\{x \in K$ such that $\rho(x)<t\}$.

Proof. This lemma follows from Sandier-Serfaty [16].

In order to bound $b(t)$ we use the following variation of a covering argument developed in [16]. In our case we have a modified regime of radii in which our function $f(r, R)$ is increasing and takes into account the limiting behavior of $\ell$.

Lemma 5.5. Assume $V \subset \mathbb{R}^{2}$ is open and $\omega \subset \mathbb{R}^{2}$ is compact. Assume $v: V \backslash \omega \rightarrow \mathbb{S}^{1}$ and $A: V \rightarrow \mathbb{R}^{2}$. Then for any $\sigma \geq r(\omega)$ such that $\sigma \leq \min \left\{1, \frac{4}{3 \ell}, \frac{1}{3 \sqrt{h_{e x}}}\right\}$ there exists a family $\left\{B_{i}\right\}$ of disjoint disks of radii $r_{i}$ such that

(1) $\sum r_{i}=\sigma$

(2) $\omega \subset \cup \bar{B}_{i} \subset \Omega_{1}$

(3) Letting $h=\operatorname{curl} A$ and $v=e^{i \varphi}$, then

$$
\frac{1}{2} \int_{B_{i} \backslash \omega}|\nabla \varphi-A|^{2}+\frac{1}{2 \ell^{2}} \int_{B_{i}}\left|h-h_{e x}\right|^{2} \geq \pi\left|d_{i}\right|\left(\log \frac{\min \left\{1, \frac{1}{\ell}, \frac{1}{\sqrt{h_{e x}}}\right\}}{r\left(B_{i}\right)}-C\right)^{+}
$$

where $d_{i}$ is the winding number of $v$ restricted to $\partial B_{i}$, if $\bar{B}_{i} \Subset \Omega_{1} \cap V$, and zero otherwise.

The proof of Lemma 5.5 follows from the two lemmas below. The first lemma takes full advantage of $\frac{r}{\ell} \int_{B_{r}} h^{2} d x$ term in the lower bound - which is not fully used in $[16,15]$, see inequality $(5.12)$.

Lemma 5.6. Let $v: B_{R} \backslash \bar{B}_{r} \rightarrow \mathbb{S}^{1}$ and $A: B_{R} \rightarrow \mathbb{R}^{2}$. Then for all $h_{e x}>0$ and $0<r<R<\min \left\{\frac{1}{2}, \frac{1}{\ell}\right\}$,

$$
\begin{aligned}
\frac{1}{2} \int_{B_{R} \backslash \bar{B}_{r}}|\nabla \varphi-A|^{2}+\frac{R-r}{2} \frac{1}{\ell} \int_{B_{r}}\left|h-h_{e x}\right|^{2} \\
\quad \geq \pi|d|\left(\log \frac{R}{r}-\frac{\ell}{2}(R-r)-\frac{h_{e x}}{2}\left(R^{2}-r^{2}\right)\right)
\end{aligned}
$$

where $d$ is the winding number of $v=e^{i \varphi}$ restricted to any circle $\partial B_{s}, r<s<R$. Furthermore, the function

$$
f(r, R)=\log \frac{R}{r}-\frac{\ell}{2}(R-r)-\frac{h_{e x}}{2}\left(R^{2}-r^{2}\right)
$$

is increasing in $R$ for $0<r<R \leq \min \left\{1, \frac{4}{3 \ell}, \frac{1}{3 \sqrt{h_{e x}}}\right\}$. 
Proof. This proof is based on the argument in [14]. Set

$$
g(t)=\frac{1}{2} \int_{\partial B_{t}}\left|\frac{\partial \varphi}{\partial \tau}-A \cdot \tau\right|^{2}+\frac{1}{2 \ell} \int_{B_{t}}\left|h-h_{e x}\right|^{2}
$$

then

$$
\begin{aligned}
\int_{B_{R} \backslash \overline{B_{r}}} g_{g l}(u, A) d x & \geq \frac{1}{2} \int_{B_{R} \backslash \bar{B}_{r}}|\nabla \varphi-A|^{2}+\frac{1}{2 \ell^{2}}(R-r) \int_{B_{r}}\left|h-h_{e x}\right|^{2} \\
& \geq \int_{r}^{R} g(t) d t .
\end{aligned}
$$

Let $\alpha_{t}=\int_{B_{t}} h$ then

$$
2 \pi d=\alpha_{t}-\int_{\partial B_{t}} \frac{\partial \varphi}{\partial \tau}-A \cdot \tau
$$

and so by Cauchy-Schwarz

$$
\int_{\partial B_{t}}\left|\frac{\partial \varphi}{\partial \tau}-A \cdot \tau\right|^{2} \geq \frac{1}{2 \pi t}\left(2 \pi d-\alpha_{t}\right)^{2} .
$$

Second,

so

$$
\int_{B_{t}}\left|h-h_{e x}\right|^{2} \geq \frac{1}{\pi t^{2}}\left(\alpha_{t}-\pi t^{2} h_{e x}\right)^{2}
$$

$$
g(t) \geq \frac{1}{4 \pi t}(2 \pi d-\alpha)^{2}+\frac{1}{2 \ell \pi t^{2}}\left(\alpha-\pi t^{2} h_{e x}\right)^{2}
$$

which we minimize over $\alpha$. Note that if $f(x)=\frac{1}{a}(c-x)^{2}+\frac{1}{b}(d-x)^{2}$ then the minimum occurs at $x=\frac{b c+a d}{a+b}$ or $f(x) \geq \frac{(c-d)^{2}}{a+b}$. Applying this to the above inequality yields

$$
\begin{aligned}
g(t) & \geq \frac{\left(2 \pi d-\pi t^{2} h_{e x}\right)^{2}}{4 \pi t+2 \ell^{2} \pi t^{2}}=\frac{(2 \pi d)^{2}-4 \pi^{2} d t^{2} h_{e x}+\pi^{2} t^{4} h_{e x}^{2}}{4 \pi t\left(1+\frac{\ell}{2} t\right)} \\
& \geq\left(\frac{\pi d^{2}}{t}-\pi t|d| h_{e x}\right)\left(1+\frac{\ell}{2} t\right)^{-1} .
\end{aligned}
$$

We compute the lower bound. Suppose $0<t<1$ then $0<\frac{\ell}{2} t<\frac{\ell}{2}$, and if $0<t \leq \min \left\{1, \frac{2}{\ell}\right\}$ then $0<\frac{\ell}{2} t \leq 1$. This implies

$$
\left(1+\frac{\ell}{2} t\right)^{-1} \geq 1-\frac{\ell}{2} t>0
$$

for all $0<t \leq \min \left\{\frac{1}{2}, \frac{1}{\ell}\right\}$. Therefore,

$$
g(t) \geq\left(\frac{\pi d^{2}}{t}-\pi t|d| h_{e x}\right)\left(1-\frac{\ell}{2} t\right) \geq \pi|d|\left(\frac{1}{t}-\frac{\ell}{2}-t h_{e x}\right)
$$


and so

for $0<r<R<\min \left\{\frac{1}{2}, \frac{1}{\ell}\right\}$.

$$
\int_{r}^{R} g(t) d t \geq \pi|d|\left(\log \frac{R}{r}-\frac{\ell}{2}(R-r)-\frac{h_{e x}}{2}\left(R^{2}-r^{2}\right)\right)
$$

We now show that $f(r, R)$ is increasing on the claimed interval. Set $\gamma=\frac{\ell}{4}$, then $f^{\prime}(r, x)=\frac{1}{x}-2 \gamma-h_{e x} x \geq 0$ for all $x$ up to

$$
\frac{\sqrt{\frac{4 \gamma^{2}}{h_{e x}^{2}}+\frac{4}{h_{e x}}}-\frac{2 \gamma}{h_{e x}}}{2}=\frac{\sqrt{\gamma^{2}+h_{e x}}-\gamma}{h_{e x}}=\frac{1}{\sqrt{\gamma^{2}+h_{e x}}+\gamma} .
$$

If $\gamma \geq \sqrt{h_{e x}}$, then $\gamma^{2}>h_{e x}$ and $f(x)$ is increasing up to $x=\frac{1}{\sqrt{\gamma^{2}+h_{e x}}+\gamma} \geq$ $\frac{1}{\sqrt{2 \gamma^{2}}+\gamma}=\frac{1}{\gamma} \frac{1}{\sqrt{2}+1} \geq \frac{1}{3 \gamma}$. On the other hand if $\gamma \leq \sqrt{h_{e x}}$, then $\gamma^{2} \leq h_{e x}$ and $f(x)$ is increasing up to $x=\frac{1}{\sqrt{\gamma^{2}+h_{e x}}+\gamma} \geq \frac{1}{\sqrt{2 h_{e x}}+\sqrt{h_{e x}}}=\frac{1}{\sqrt{h_{e x}}} \frac{1}{\sqrt{2}+1} \geq \frac{1}{3 \sqrt{h_{e x}}}$. Combining both estimates yields the lemma.

Finally, we complete the proof of Lemma 5.7 with the following result that performs the vortex ball growing / merging process, found in [14]. We remark the only difference in this result is the potentially smaller interval on which $f(r, \cdot)$ is increasing.

Lemma 5.7. Let $f(r, R)$ satisfy the following properties:

(1) $f(r, \cdot)$ is increasing on $\left[0, \min \left\{1, \frac{4}{3 \ell}, \frac{1}{3 \sqrt{h_{e x}}}\right\}\right]$.

(2) $f(r, r)=0$, thus $f(r, R) \geq 0$ for all $r \leq R \leq \min \left\{1, \frac{4}{3 \ell}, \frac{1}{3 \sqrt{h_{e x}}}\right\}$.

(3) $f(r, s)+f(s, R)=f(r, R)$.

(4) If $\left(0 \leq r_{i} \leq R_{i}\right)$ are $2 k$ positive real numbers and $d_{i}$ are integers, and

$$
\frac{R_{i}}{r_{i}}=\frac{R_{j}}{r_{j}}=\alpha>1
$$

for all $i, j$. Then

$$
\sum_{i=1}^{k}\left|d_{i}\right| f\left(r_{i}, R_{i}\right) \geq\left(\sum_{i=1}^{k}\left|d_{i}\right|\right) f\left(\sum_{i=1}^{k} r_{i}, \sum_{i=1}^{k} R_{i}\right) .
$$

Proof. The proof is found in [14], see also [16] for example.

We can now complete the lower bound on $b(t)$.

Proof of Lemma 5.5. This is essentially proved in [16] except for the modified regime of radii and the consideration of $\ell$ in (5.12); however, we include the argument for the sake of completeness. 
1. We first define the vortex ball growing / merging process. In particular we have two cases:

(a) If $i \neq j$ and $\overline{B_{i}} \cap \overline{B_{j}}=\varnothing$ then grow the balls at a constant rate with the same centers such that ratio $\alpha(s)$ is constant.

(b) If some $\overline{B_{1}} \cap \overline{B_{2}} \neq \varnothing$, then there is a ball $B$ such that $B_{1} \cup B_{2} \subset B$ for the family at some time $s_{0}$ and replace them with $B$. If there is a $\bar{B} \cap \overline{B_{3}} \neq \varnothing$ then enlarge $B$ so that $B_{1} \cup B_{2} \cup B_{3} \subset B$ and $r(B)=$ $r\left(B_{1}\right)+r\left(B_{2}\right)+r\left(B_{3}\right)$. Then modify $\left\{B_{i}\left(s_{0}\right)\right\}$ by removing the balls that have been merged into $B$ and replacing them by $B$. Then the seed size of $B$ is the sum of the seed sizes of $B_{1}, \ldots, B_{l}$. Indeed the $\overline{B_{1}}, \ldots, \overline{B_{l}}$ are disjoint and

$$
\frac{r(B)}{\varepsilon(B)}=\frac{\sum_{i=1}^{l} r\left(B_{i}\right)}{\sum_{i=1}^{l} \varepsilon\left(B_{i}\right)}=\alpha\left(s_{0}\right)
$$

and so the modified family satisfies the rules. The left over balls in the family satisfy the criteria, and we inflate the balls via Case 1.

2. Now let $d\left(B_{i}(s)\right)$ be the degree of $v$ restricted to $\partial B_{i}(s)$ if $\overline{B_{i}(s)} \Subset V$ and zero otherwise then we claim

$$
\begin{aligned}
\frac{1}{2} \int_{B_{i}(s) \backslash \omega}|\nabla \varphi-A|^{2}+\frac{1}{2 \ell^{2}} \int_{B_{i}(s)}\left|h-h_{e x}\right|^{2} \\
\geq \frac{1}{2} \int_{B_{i}(s) \backslash \omega}|\nabla \varphi-A|^{2}+\frac{r\left(B_{i}(s)\right)}{2 \ell} \int_{B_{i}(s)}\left|h-h_{e x}\right|^{2} \\
\geq\left|d\left(B_{i}(s)\right)\right| f\left(\varepsilon\left(B_{i}(s)\right), r\left(B_{i}(s)\right)\right) .
\end{aligned}
$$

To establish (5.12) we remark the first inequality follows trivially since $r\left(B_{i}(s)\right) \leq$ $\sigma \leq \frac{1}{\ell}$. Next, we establish the second inequality; note that when $s=0$, $\varepsilon\left(B_{i}(0)\right)=r\left(B_{i}(0)\right)$ and $f(s, s)=0$. We check that (5.12) holds through the growth process. Suppose the inequality holds for $B=B_{i}(s)$ and grows to $B^{\prime}=B_{i}(t)$ for $t>s$ and $B^{\prime} \Subset V$. Then $d\left(B^{\prime}\right)=d(B), \varepsilon\left(B^{\prime}\right)=\varepsilon(B)$ and

$$
\begin{aligned}
& \frac{1}{2} \int_{B^{\prime} \backslash \omega}|\nabla \varphi-A|^{2}+\frac{r\left(B^{\prime}\right)}{2 \ell} \int_{B^{\prime}}\left|h-h_{e x}\right|^{2} \\
& \geq \frac{1}{2} \int_{B \backslash \omega}|\nabla \varphi-A|^{2}+\frac{r(B)}{2 \ell} \int_{B^{\prime}}\left|h-h_{e x}\right|^{2} \\
& \quad \frac{1}{2} \int_{B^{\prime} \backslash B}|\nabla \varphi-A|^{2}+\frac{r\left(B^{\prime}\right)-r(B)}{2 \ell} \int_{B^{\prime}}\left|h-h_{e x}\right|^{2} \\
& \geq|d| f(\varepsilon(B), r(B))+|d| f\left(r(B), r\left(B^{\prime}\right)\right)=|d| f\left(\varepsilon\left(B^{\prime}\right), r\left(B^{\prime}\right)\right)
\end{aligned}
$$


On the other hand if we have a merging, we see that for balls $\left\{B_{i}\right\}_{i=1}^{l}$ merged into $B$ we have

$$
\begin{aligned}
& \frac{1}{2} \int_{B \backslash \omega}|\nabla \varphi-A|^{2}+\frac{r\left(B^{\prime}\right)}{2 \ell} \int_{B}\left|h-h_{e x}\right|^{2} \\
& \geq \sum_{i=1}^{l} \frac{1}{2} \int_{B_{i} \backslash \omega}|\nabla \varphi-A|^{2}+\frac{r\left(B_{i}\right)}{2 \ell} \int_{B_{i}}\left|h-h_{e x}\right|^{2} \\
& \geq \sum_{i=1}^{l}\left|d_{i}\right| f\left(\varepsilon\left(B_{i}\right), r\left(B_{i}\right)\right) \\
& \geq\left(\sum_{i=1}^{l}\left|d\left(\varepsilon\left(B_{i}\right)\right)\right|\right) f\left(\sum_{i=1}^{l} \varepsilon\left(B_{i}\right), \sum_{i=1}^{l} r\left(B_{i}\right)\right) \\
& \geq|d(B)| f(\varepsilon(B), r(B)) .
\end{aligned}
$$

3. Finally, using (5.12) we grow the balls until $\sum_{i} r\left(B_{i}\right)=\sigma$. Since $\omega \subset$ $\cup B_{i}(0) \subset B_{i}(s)$ and $\sigma<\min \left\{1, \frac{1}{2 \ell}, \frac{1}{2 \sqrt{h_{e x}}}\right\}$, then $r\left(B_{i}(s)\right) \leq \min \left\{1, \frac{1}{2 \ell}, \frac{1}{2 \sqrt{h_{e x}}}\right\}$. Hence

$$
f\left(\varepsilon\left(B_{i}(s)\right), r\left(B_{i}(s)\right)\right) \geq\left(\log \frac{r\left(B_{i}(s)\right)}{\varepsilon\left(B_{i}(s)\right)}-C\right)_{+} .
$$

Since $\frac{r\left(B_{i}(s)\right)}{\varepsilon\left(B_{i}(s)\right)}=\frac{\sum_{i} r\left(B_{i}(s)\right)}{\sum_{i} \varepsilon\left(B_{i}(s)\right)}=\frac{\sigma}{r(\omega)}$, then using (5.12) we have for all $\overline{B_{i}} \Subset V$

$$
\int_{B_{i} \backslash \omega}|\nabla \varphi-A|^{2}+\frac{1}{2 \ell^{2}} \int_{B_{i}}\left|h-h_{e x}\right|^{2} \geq\left|d_{i}\right|\left(\log \frac{\sigma}{r(\omega)}-C\right)_{+},
$$

which proves the lemma.

We use $|\log \varepsilon| \ll h_{e x}$ implicitly at many steps in the following

Lemma 5.8. Let $K$ be the square chosen in Lemma 5.4 with sidelength $\delta$ and recall $\Omega_{t}=\{x \in K$ such that $\rho(x)<t\}$. Then for all $0<t<1$

$$
b(t) \geq \pi\left(\sum_{i: B_{i} \Subset K}\left|d_{B_{i}}\right|\right)\left(\log \frac{1}{r\left(\Omega_{t}\right) \max \left\{\ell, \sqrt{h_{e x}}\right\}}-C\right)^{+}
$$

and

$$
2 \pi d_{t} \geq h_{e x} \delta^{2}\left[\left(1-\frac{\alpha}{\delta}\right)^{2}-\frac{\chi}{t} \sqrt{\frac{\delta}{\alpha}}-\frac{2 \chi}{t} \sqrt{\delta^{2} \ell^{2}}\right]
$$

where $\chi^{2}=\frac{G_{K}}{h_{e x}^{2} \delta^{4}}$ and $\min \left\{\frac{1}{\ell}, \frac{1}{\sqrt{h_{e x}}}\right\} \leq \alpha \leq \frac{\delta}{4}$. 
Proof. If we take $\omega=\Omega_{t}, v=\frac{u}{|u|}, V=K$ and $\sigma=\min \left\{1, \frac{4}{3 \ell}, \frac{1}{3 \sqrt{h_{e x}}}\right\}$ then Lemma 5.5 and Lemma 5.7 imply

$$
b(t) \geq \pi\left(\sum_{i: B_{i} \Subset K}\left|d_{B_{i}}\right|\right)\left(\log \frac{\min \left\{1, \frac{1}{\ell}, \frac{1}{\sqrt{h_{e x}}}\right\}}{r\left(\Omega_{t}\right)}-C\right)^{+}
$$

and the difficulty comes in controlling $\left(\sum_{i: B_{i} \Subset K}\left|d_{B_{i}}\right|\right)$. Here the interaction between magnetic field and degree is nontrivial. We now provide an estimate on the degree via energy bounds.

Step 1. Consider the following minimization problem:

$$
\min _{A \in H^{1}\left(K, \mathbb{R}^{2}\right) \operatorname{div}} A=0 \frac{1}{2} \int_{K \backslash \bar{\Omega}_{t}}|\nabla \varphi-A|^{2} d x+\frac{1}{2 \ell^{2}} \int_{K}\left|\operatorname{curl} A-h_{e x}\right|^{2} d x
$$

where $\varphi=\arg u$. The minimizer is achieved for some $\bar{A}$ with $\bar{h}=\operatorname{curl} \bar{A}$, and it satisfies

$$
-\frac{1}{\ell^{2}} \nabla^{\perp} \bar{h}=\nabla \varphi-\bar{A} \text { in } K \backslash \bar{\Omega}_{t}
$$

with $\bar{h}=h_{e x}$ on $\partial K$ and $\bar{h}=$ constant in $\bar{\Omega}_{t}$. On each component $\omega_{i}$ of $\Omega_{t}$ we compute the degree around $\partial \omega_{i}$,

$$
\begin{aligned}
2 \pi d_{i} & =\int_{\partial \omega_{i}} \frac{\partial \varphi}{\partial \tau} d l=\int_{\partial \omega_{i}} \bar{A} \cdot \tau-\frac{1}{\ell^{2}} \tau \cdot \nabla^{\perp} \bar{h} d l \\
& =\int_{\omega_{i}} \bar{h} d x-\frac{1}{\ell^{2}} \int_{\partial \omega_{i}} \frac{\partial \bar{h}}{\partial n} d l .
\end{aligned}
$$

Furthermore, in $K \backslash \bar{\Omega}_{t}$ then

$$
-\frac{1}{\ell^{2}} \Delta \bar{h}+\bar{h}=0
$$

and so for some $K^{\prime} \subset K$ we have

$$
\begin{aligned}
-\frac{1}{\ell^{2}} \int_{\partial K^{\prime}} \frac{\partial \bar{h}}{\partial n} d l+\int_{K^{\prime} \backslash \bar{\Omega}_{t}} \bar{h} & =-\sum_{i} \frac{1}{\ell^{2}} \int_{\partial \omega_{i}} \frac{\partial \bar{h}}{\partial n} d l \\
& =-\sum_{i} \int_{\omega_{i}} \bar{h} d x+2 \pi \sum_{i: \omega_{i} \subset K^{\prime}} d_{i}
\end{aligned}
$$

or

$$
2 \pi \sum_{i: \omega_{i} \subset K^{\prime}} d_{i}=-\frac{1}{\ell^{2}} \int_{\partial K^{\prime}} \frac{\partial \bar{h}}{\partial n} d l+\int_{K^{\prime}} \bar{h} d x
$$

which controls the total degree by the magnetic field. 
Step 2. Consider now for $|u| \geq t$ then $\nabla \varphi-\bar{A}=-\frac{1}{\ell^{2}} \nabla^{\perp} \bar{h}$ and

$$
\begin{aligned}
G_{K} & \geq \frac{t^{2}}{2}\left[\int_{K \backslash \Omega_{t}}|\nabla \varphi-\bar{A}|^{2}+\frac{1}{\ell^{2}} \int_{K}\left|\bar{h}-h_{e x}\right|^{2} d x\right] \\
& \geq \frac{t^{2}}{2 \ell^{2}} \int_{K} \frac{1}{\ell^{2}}|\nabla \bar{h}|^{2}+\left|\bar{h}-h_{e x}\right|^{2} d x,
\end{aligned}
$$

then

$$
\int_{K} \frac{1}{\ell^{2}}|\nabla \bar{h}|^{2}+\left|\bar{h}-h_{e x}\right|^{2} \leq \frac{2 \ell^{2}}{t^{2}} G_{K}
$$

Set $K_{t}=\{x \in K: \operatorname{dist}(x, \partial K)>t\}$ then for any $\min \left\{\frac{4}{3 \ell}, \frac{1}{3 \sqrt{h_{e x}}}\right\} \leq \alpha \leq \frac{\delta}{4}$, the set $T=\left\{t \in[0, \alpha]\right.$ such that $\left.\partial K_{t} \cap \cup_{i} B_{i}=\varnothing\right\}$ has measure at least $\frac{\alpha}{2}$ since $\sum r_{i}=\min \left\{\frac{4}{3 \ell}, \frac{1}{3 \sqrt{h_{e x}}}\right\}$. If we define the set

$$
T^{\prime}=\left\{t \in T \text { such that } \int_{\partial K_{t}}\left|\frac{\partial \bar{h}}{\partial n}\right|^{2} d l \leq \frac{2 \ell^{2}}{\alpha t^{2}} G_{K}\right\}
$$

then by the energy estimate

$$
\frac{1}{\ell^{2}} \int_{0}^{\alpha} \int_{\partial K_{t}}|\nabla \bar{h}|^{2} d l d t=\frac{1}{\ell^{2}} \int_{K \backslash \bar{K}_{\alpha}}|\nabla \bar{h}|^{2} \leq \frac{2 \ell^{2}}{t^{2}} G_{K}
$$

implies there exists $t_{0} \in(0, \alpha)$ such that $0 \leq t_{0} \leq \alpha<\frac{\delta}{4}$ with $\partial K_{t_{0}} \cap \bigcup B_{i}=\varnothing$ and $\frac{1}{\ell^{2}} \int_{\partial K_{t_{0}}}\left|\frac{\partial \bar{h}}{\partial n}\right|^{2} d l<\frac{2 \ell^{2} G_{K}}{\alpha t^{2}}$. Thus

$$
\begin{aligned}
2 \pi d_{t} & \geq 2 \pi \sum_{i} d_{B_{i}}=-\frac{1}{\ell^{2}} \int_{\partial K_{t_{0}}} \frac{\partial \bar{h}}{\partial n} d l+\int_{K_{t_{0}}} \bar{h} d x \\
& \geq \int_{K_{t_{0}}} h_{e x} d x-\left|\int_{K_{t_{0}}} \bar{h}-h_{e x} d x\right|-\left|\frac{1}{\ell^{2}} \int_{\partial K_{t_{0}}} \frac{\partial \bar{h}}{\partial n} d l\right| .
\end{aligned}
$$

Naively estimating the last two terms by

$$
\left|\int_{K_{t_{0}}} \bar{h}-h_{e x} d x\right| \leq\left\|\bar{h}-h_{e x}\right\|_{L^{2}\left(K_{t_{0}}\right)}\left|K_{t_{0}}\right|^{\frac{1}{2}} \leq \sqrt{\frac{G_{K} \delta^{2} \ell^{2}}{t^{2}}}
$$

and

$$
\left|\frac{1}{\ell^{2}} \int_{\partial K_{t_{0}}} \frac{\partial \bar{h}}{\partial n} d x\right| \leq\left\|\frac{1}{\ell^{2}} \frac{\partial \bar{h}}{\partial n}\right\|_{L^{2}\left(K_{t_{0}}\right)}\left|\partial K_{t_{0}}\right|^{\frac{1}{2}} \leq \sqrt{\frac{G_{K} \delta}{\alpha t^{2}}}
$$

yields

$$
2 \pi d_{t} \geq h_{e x} \delta^{2}\left[\left(1-\frac{\alpha}{\delta}\right)^{2}-\frac{1}{t} \sqrt{\frac{G_{K}}{h_{e x}^{2} \delta^{4}} \frac{\delta}{\alpha}}-\frac{1}{t} \sqrt{\frac{G_{K}}{h_{e x}^{2} \delta^{4}} \ell^{2} \delta^{2}}\right]
$$


Remark 5.9. In order to use (5.14) we should have the following estimates hold

$$
\frac{\alpha}{\delta} \ll 1 \quad \delta \ll 1 \quad \chi \ll 1 \quad \frac{\chi^{2} \delta}{\alpha} \ll 1 \quad \chi \delta \ell \ll 1,
$$

otherwise the right hand side of (5.14) is zero, and the lower bound fails. Note that (5.16) implies the rate $\frac{\delta}{\alpha} \ll \frac{1}{\chi^{2}}$, which we use in Lemma 5.10 to choose $\alpha$ correctly.

We are now able to proof the essential lower bound on the square.

Lemma 5.10. Suppose (5.5) and (5.7) hold, then

$$
G_{K} \geq \frac{1}{2} h_{e x} \delta^{2} V_{\varepsilon}\left(1-o_{\varepsilon}(1)\right) .
$$

Proof. This lower bound is established via estimates in the spirit of [16].

Step 1. We examine $a(t)+2 t b(t)$, using (5.11) and (5.13), and note the sum is of the form $C_{1} r+C_{2}\left(\log \frac{C_{3}}{r}-C_{4}\right)^{+}$for $r=r\left(\Omega_{t}\right)$. The function $p(r)=$ $C_{1} r+C_{2}\left(\log \frac{C_{3}}{r}-C_{4}\right)^{+}$has critical points at $r=\left\{\frac{C_{2}}{C_{1}}, C_{3} e^{-C_{4}}\right\}$. Therefore, $p(r)$ satisfies

$$
p(r) \geq \min \left\{\frac{C_{1} C_{3}}{e^{C_{4}}}, C_{2}+C_{2}\left(\log \frac{C_{1} C_{3}}{C_{2}}-C_{4}\right)^{+}\right\}
$$

and since $x \mapsto-\log c x$ is decreasing in $x$, we have

$$
p(r) \geq C_{2}\left(\log \frac{C_{1} C_{3}}{C_{2}}-C_{4}\right)^{+} .
$$

We apply to our lower bound with

$$
\begin{aligned}
& C_{1}=\frac{\left(1-t^{2}\right)}{\varepsilon}, \quad C_{2}=h_{e x} \delta^{2}\left(\left(1-\frac{\alpha}{\delta}\right)^{2} t-\chi \min \left\{\sqrt{\frac{\delta}{\alpha}}, \delta \ell\right\}\right)^{+}, \\
& C_{3}=\min \left\{\frac{1}{\ell}, \frac{1}{\sqrt{h_{e x}}}\right\}, \quad C_{4}=C .
\end{aligned}
$$

¿From Remark 5.9 if (5.16) is satisfied then $C_{2}$ is nontrivial. From the lower bound $(x-a)^{+}(y-b)^{+} \geq x y-x b-y a$ we have

$$
\begin{aligned}
a(t)+ & 2 t b(t) \\
\geq & -C_{2} \log C_{2} \\
& +h_{e x} \delta^{2}\left(1-\frac{\alpha}{\delta}\right)^{2}\left[t \log \left|1-t^{2}\right|+t \log \left(\frac{1}{\varepsilon \max \left\{\ell, \sqrt{h_{e x}}\right\}}\right)-C t\right] \\
& -\chi \min \left\{\sqrt{\frac{\delta}{\alpha}}, \delta \ell\right\}\left[\log \left|1-t^{2}\right|+\log \left(\frac{1}{\varepsilon \max \left\{\ell, \sqrt{h_{e x}}\right\}}\right)\right] .
\end{aligned}
$$


First note that $-C_{2} \log C_{2} \geq-C_{2} \log h_{e x} \delta^{2}$ since

$$
0 \leq\left(\left(1-\frac{\alpha}{\delta}\right)^{2} t-\chi \min \left\{\sqrt{\frac{\delta}{\alpha}}, \delta \ell\right\}\right)^{+} \leq 1
$$

Furthermore, since $1 \ll h_{e x} \delta^{2}$, hence $-C_{2} \log C_{2} \geq-h_{e x} \delta^{2} \log \left(h_{e x} \delta^{2}\right)$. We integrate the above lower bound. Using $\left|\int_{0}^{1} t \log \right| 1-t^{2}|d t|=6 \log 2-4$ and $\left|\int_{0}^{1} \log \right| 1-t^{2}|d t|=2 \log 2-2$, we find

$$
\begin{aligned}
\int_{\frac{\varepsilon}{\delta}}^{1} a(t) & +2 t b(t) d t \\
\geq & \frac{1}{2}\left(1-\frac{\alpha}{\delta}\right)^{2} h_{e x} \delta^{2} \log \left(\frac{1}{\varepsilon \max \left\{\ell, \sqrt{h_{e x}}\right\}}\right) \\
& -\chi \min \left\{\sqrt{\frac{\delta}{\alpha}}, \delta \ell\right\} \log \left(\frac{1}{\varepsilon \max \left\{\ell, \sqrt{h_{e x}}\right\}}\right)-h_{e x} \delta^{2} \log \left(h_{e x} \delta^{2}\right) \\
& -C\left[\chi \min \left\{\sqrt{\frac{\delta}{\alpha}}, \delta \ell\right\}+h_{e x} \delta^{2}\right] .
\end{aligned}
$$

We now show that most of the terms in (5.19) are $o_{\varepsilon}(1)$ compared to the leading order term $\frac{1}{2} h_{e x} \delta^{2} V_{\varepsilon}$.

Step 2. We proceed with several comparison arguments that will be grouped together at the end of this step. ¿From (5.5) we see $h_{e x} \delta^{2} \ll V_{\varepsilon}^{2}$, hence $\log \left(h_{e x} \delta\right) \ll C \log V_{\varepsilon} \ll V_{\varepsilon}$ since $V_{\varepsilon} \gg 1$. Therefore,

$$
h_{e x} \delta^{2} \log \left(h_{e x} \delta^{2}\right) \ll h_{e x} \delta^{2} V_{\varepsilon}
$$

Next from (5.1) we have

$$
\frac{V_{\varepsilon} \ell^{2}}{h_{e x}} \leq \frac{V_{\varepsilon}}{h_{e x}} \max \left\{\ell^{2}, 1\right\} \ll 1 .
$$

Recall that $\chi^{2}=\frac{G_{K}}{h_{e x}^{2} \delta^{4}} ;$ therefore,

$$
\delta^{2} \chi^{2} \ell^{2}=\frac{G_{K} \ell^{2}}{\delta^{2} h_{e x}^{2}} \leq C \frac{h_{e x} V_{\varepsilon} \ell^{2}}{h_{e x}^{2}}=C \frac{V_{\varepsilon} \ell^{2}}{h_{e x}} \ll C,
$$

follows from (5.7) and (5.21).

Note that

$$
\chi^{2}=\frac{G_{K}}{h_{e x}^{2} \delta^{4}} \leq \frac{V_{\varepsilon}}{h_{e x} \delta^{2}} \ll 1
$$


where we used (5.5), hence $\chi \rightarrow 0$. ¿From Remark 5.9 we choose $\alpha=\delta \chi^{2 \gamma}$ for $0<\gamma<\frac{1}{2}$, then (5.23) implies both

$$
\frac{\alpha}{\delta}=\chi^{2 \gamma} \ll 1
$$

and

$$
\frac{\chi^{2} \delta}{\alpha}=\chi^{2(1-\gamma)} \ll 1
$$

Since

$$
1 \ll V_{\varepsilon} \ll h_{e x} \delta^{2}
$$

then using (5.19), (5.20), (5.22), (5.24), (5.25), (5.26), we establish the lower bound at leading order:

$$
\int_{\frac{\varepsilon}{\delta}}^{1} a(t)+2 t b(t) d t \geq \frac{1}{2} h_{e x} \delta^{2} V_{\varepsilon}\left(1-o_{\varepsilon}(1)\right) .
$$

Using Step 1 then (5.27) becomes

$$
\int_{0}^{1} a(t)+2 t b(t) d t \geq \int_{\frac{\varepsilon}{\delta}}^{1} a(t)+2 t b(t) d t \geq \frac{1}{2} h_{e x} \delta^{2} V_{\varepsilon}\left(1-o_{\varepsilon}(1)\right),
$$

and hence (5.17).

Proof of Proposition 5.1. We now consider the energy $G_{\varepsilon}$ in the entire domain. From Lemma 5.10 and (5.8)

$$
\begin{aligned}
G_{\varepsilon}\left(u_{\varepsilon}, A_{\varepsilon}\right) & \geq \sum_{K_{i} \subset U}\left[\sum_{G_{K_{i}} \leq h_{e x} \delta^{2} V_{\varepsilon}} G_{K_{i}}+\sum_{G_{K_{i}}>h_{e x} \delta^{2} V_{\varepsilon}} G_{K_{i}}\right] \\
& \geq \sum_{K_{i} \subset U}\left[\sum_{G_{K_{i}} \leq h_{e x} \delta^{2} V_{\varepsilon}} \frac{1}{2} h_{e x} \delta^{2} V_{\varepsilon}\left(1-o_{\varepsilon}(1)\right)+\sum_{G_{K_{i}}>h_{e x} \delta^{2} V_{\varepsilon}} \frac{1}{2} h_{e x} \delta^{2} V_{\varepsilon}\right] \\
& \geq \sum_{K_{i} \subset U} \frac{1}{2} h_{e x} \delta^{2} V_{\varepsilon}\left(1-o_{\varepsilon}(1)\right) \\
& \geq \frac{1}{2}|U| h_{e x} V_{\varepsilon}\left(1-o_{\varepsilon}\right),
\end{aligned}
$$

since for $\varepsilon$ small, $\sum_{K_{i} \subset U} \delta^{2} \geq|U|\left(1-o_{\varepsilon}\right)$.

\section{REFERENCES}

[1] Aftalion, A. and Dancer, E. N. On the symmetry and uniqueness of solutions of the Ginzburg-Landau equations for small domains. Commun. Contemp. Math. 3 (2001), 114.

[2] Aftalion, A., Du, Q. The bifurcation diagrams for the Ginzburg-Landau system of superconductivity. Physica D 163 (2002), 94-105. 
[3] Bethuel, F., Brezis, H. and Hélein, F. Ginzburg-Landau Vortices Birkhäuser, Boston, 1994.

[4] Bethuel, F. and Riviere, T. Vortices for a variational problem related to superconductivity Ann. Inst. H. Poincar Anal. Non Linaire 12 (1995), 243-303.

[5] Brezis, H. and Serfaty, S. A variational formulation for the two-sided obstacle problem with measure data. Comm. Contemp. Math. 4 (2002), 357-374.

[6] Friedman, A. Variational principles and free-boundary problems.John Wiley \& Sons, Inc., New York, 1982

[7] Jerrard, R. L. Lower bounds for generalized Ginzburg-Landau functionals, SIAM J. Math. Anal. 30 (1999), 721-746.

[8] Jerrard, R. L. and Soner, H. M. The Jacobian and the Ginzburg-Landau energy. Calc. Var. Partial Differential Equations 14 (2002), 151-191.

[9] Jerrard, R. L. and Soner, H. M. Limiting behavior of the Ginzburg-Landau functional. J. Funct. Anal. 192 (2002), 524-561.

[10] Jerrard, R. L. and Spirn, D. Refined Jacobian estimates and the Ginzburg-Landau energy, Indiana Univ. Math. J. 56 (2007), 135-186.

[11] Kurzke, M. and Spirn, D. Gamma limit of the nonself-dual Chern-Simons-Higgs energy, submitted.

[12] Kurzke, M. and Spirn, D. Scaling limits of the Chern-Simons-Higgs energy, submitted.

[13] Kurzke, M. and Spirn, D. On the energy of a Chern-Simons-Higgs vortex lattice, submitted.

[14] Sandier, E. Lower bounds for the energy of unit vector fields and applications J. Funct. Anal. 152 (1998), 379-403.

[15] Sandier, E. and Serfaty, S. Vortices in the Magnetic Ginzburg-Landau Model. Progress in Nonlinear Differential Equations and Their Applications 70. Birkhäuser, Boston 2007.

[16] Sandier, E. and Serfaty, S. On the energy of type-II superconductors in the mixed phase. Rev. Math. Phys. 12 (2000), 1219-1257.

[17] Sandier, E., and Serfaty, S. A rigorous derivation of a free boundary problem arising in superconductivity. Ann. Sci. École Norm. Sup. (4) 33 (2000), 561-592.

[18] Sandier, E. and Serfaty, S. Global Minimizers for the Ginzburg-Landau functional below the first critical field.Ann. Inst. H. Poincaré Anal. Non Linéaire 17 (2000), 119-145.

[19] Serfaty, S. Local minimizers for the Ginzburg-Landau energy near critical magnetic field. I. Commun. Contemp. Math. 1 (1999), 213-254.

[20] Serfaty, S. Local minimizers for the Ginzburg-Landau energy near critical magnetic field. II. Commun. Contemp. Math. 1 (1999), 295-333.

[21] Spirn, D. Vortex motion law for the Schrödinger-Ginzburg-Landau equations. SIAM J. Math. Anal. 34 (2003), 1435-1476.

[22] Yang, Y. Solitons in field theory and nonlinear analysis. Springer Monographs in Mathematics. Springer-Verlag, New York, 2001.

Department of Mathematics, Humboldt University, Berlin, Germany

E-mail address: kurzkem@mathematik.hu-berlin.de

School of Mathematics, University of Minnesota, Minneapolis, MN 55455

E-mail address: spirn@math.umn.edu 